\title{
Genomic and transcriptomic analysis of the thermophilic lignocellulose-degrading fungus Thielavia terrestris LPH172
}

\author{
Monika Tõlgo ${ }^{1,2 \dagger}$, Silvia Hüttner ${ }^{1,2 \dagger}$, Peter Rugbjerg ${ }^{2}$, Nguyen Thanh Thuy ${ }^{3}, V_{u}$ Nguyen Thanh ${ }^{3}$,
} Johan Larsbrink ${ }^{1,2}$ and Lisbeth Olsson ${ }^{1,2^{*}}$ (1)

\begin{abstract}
Background: Biomass-degrading enzymes with improved activity and stability can increase substrate saccharification and make biorefineries economically feasible. Filamentous fungi are a rich source of carbohydrate-active enzymes (CAZymes) for biomass degradation. The newly isolated LPH172 strain of the thermophilic Ascomycete Thielavia terrestris has been shown to possess high xylanase and cellulase activities and tolerate low pH and high temperatures. Here, we aimed to illuminate the lignocellulose-degrading machinery and novel carbohydrate-active enzymes in LPH172 in detail.
\end{abstract}

Results: We sequenced and analyzed the 36.6-Mb genome and transcriptome of $\mathrm{LPH} 172$ during growth on glucose, cellulose, rice straw, and beechwood xylan. 10,128 predicted genes were found in total, which included 411 CAZy domains. Compared to other fungi, auxiliary activity (AA) domains were particularly enriched. A higher GC content was found in coding sequences compared to the overall genome, as well as a high GC3 content, which is hypothesized to contribute to thermophilicity. Primarily auxiliary activity (AA) family 9 lytic polysaccharide monooxygenase (LPMO) and glycoside hydrolase (GH) family 7 glucanase encoding genes were upregulated when LPH172 was cultivated on cellulosic substrates. Conventional hemicellulose encoding genes (GH10, GH11 and various CEs), as well as AA9 LPMOs, were upregulated when LPH172 was cultivated on xylan. The observed co-expression and co-upregulation of genes encoding AA9 LPMOs, other AA CAZymes, and (hemi)cellulases point to a complex and nuanced degradation strategy.

Conclusions: Our analysis of the genome and transcriptome of T. terrestris LPH172 elucidates the enzyme arsenal that the fungus uses to degrade lignocellulosic substrates. The study provides the basis for future characterization of potential new enzymes for industrial biomass saccharification.

Keywords: Biomass degradation, Carbohydrate active enzymes, Cellulose, Filamentous fungi, LPMO, Thermostable enzymes, Transcriptome, Xylan

*Correspondence: lisbeth.olsson@chalmers.se

${ }^{\dagger}$ Monika Tõlgo and Silvia Hüttner share first authorship

${ }^{1}$ Wallenberg Wood Science Centre, Department of Biology and Biological

Engineering, Chalmers University of Technology, SE-412 96 Gothenburg, Sweden

Full list of author information is available at the end of the article

\section{Background}

The biorefinery concept represents the basis for a more sustainable bio-based economy aimed at converting abundant renewable biomass sources into energy and value-added products. Today, around 40 lignocellulosic biorefineries operate across Europe [1]. Even though lignocellulose is a potential biomass resource, its degradation is impeded by high lignin content and heterogeneity

c) The Author(s) 2021. This article is licensed under a Creative Commons Attribution 4.0 International License, which permits use, sharing, adaptation, distribution and reproduction in any medium or format, as long as you give appropriate credit to the original author(s) and the source, provide a link to the Creative Commons licence, and indicate if changes were made. The images or other third party material in this article are included in the article's Creative Commons licence, unless indicated otherwise in a credit line to the material. If material is not included in the article's Creative Commons licence and your intended use is not permitted by statutory regulation or exceeds the permitted use, you will need to obtain permission directly from the copyright holder. To view a copy of this licence, visit http://creativeco mmons.org/licenses/by/4.0/. The Creative Commons Public Domain Dedication waiver (http://creativecommons.org/publicdomain/ zero/1.0/) applies to the data made available in this article, unless otherwise stated in a credit line to the data. 
of its polysaccharide constituents [2,3]. Biomass saccharification into fermentable monomeric sugars by enzymatic hydrolysis is a crucial step in a biorefinery, but it is hindered by the high cost of enzymes. Indeed, enzymes have been estimated to add $1 \mathrm{USD} /$ gallon to the cost of bioethanol produced from poplar. Thus, there is strong demand for improved enzyme activity and stability [4].

Various potential industrial enzymes exist in nature [5] and the Kingdom Fungi, with more than a million species, represents a particularly rich source [6]. As major biomass degraders, fungi possess a broad array of enzymes suitable for lignocellulose degradation, which are often secreted in large quantities [7]. Thermophilic and thermo-tolerant fungi are especially interesting, as their enzymes can usually endure harsh conditions used in the industry, such as extreme temperatures or $\mathrm{pH}$ and harsh solvents $[8,9]$. For example, biomass hydrolysis by the industrial Trichoderma reesei enzymes in a separate hydrolysis-fermentation process (SHF), is performed at $45-50{ }^{\circ} \mathrm{C}$ and $\mathrm{pH} 5$ and therefore additional enzymes that are added to this process to enhance hydrolysis further should show high activity under the same conditions. Thermostable enzymes can lower industrial processing costs as they can achieve faster reaction rates, greater stability, and are more easily adjustable to various setups [10]. Yet, it should be kept in mind that novel enzymes from thermophiles are not necessarily thermostable when produced heterologously [11].

Thielavia terrestris (syn Thermothielavioides terrestris [12]) is a well-known filamentous fungus identified in 1983 as a potential source of thermostable industrial enzymes based on successful (hemi)cellulase assays [13, 14]. The species is a thermophilic saprobic Ascomycete isolated mainly from soil and compost in Asia [15-17] and from a cave cricket species in North America [18]. $T$. terrestris also played a pivotal role in the discovery of the cellulase-boosting effect of the glycoside hydrolase family 61 (GH61) proteins [19-23], today known as auxiliary activity family 9 (AA9) lytic polysaccharide monooxygenases (LPMOs) [24]. As described by Merino and Cherry [19], cultivation broth from T. terrestris primed for cellulase production showed striking synergy in degrading pre-treated corn stover when supplemented with the enzyme cocktail Celluclast. In 2011, T. terrestris strain NRRL 8126 and Myceliophthora thermophila ATCC 42464 were the first thermophiles whose genomes were fully sequenced and the first filamentous fungi with known telomere-to-telomere genome sequences [25]. The same study showed that $T$. terrestris could potentially degrade all plant cell wall polysaccharides and the fungus hydrolyzed alfalfa straw at temperature optima of $40{ }^{\circ} \mathrm{C}$ and $60{ }^{\circ} \mathrm{C}$. As shown by proteomics analyses [26] and detailed biochemical characterization [15, 16, 20, 27-33],
T. terrestris produces an array of biomass-degrading enzymes. However, no study has elucidated the gene expression mechanisms underlying the lignocellulolytic machinery of the fungus in detail.

In recent years, it has become clear that genetic or gene expression differences between fungal strains of the same species are not uncommon [17, 34-37]. In this study, we set out to sequence and analyze the genome and transcriptome of the newly isolated $T$. terrestris strain LPH172, which is characterized by superior enzymatic activity, thermostability, and $\mathrm{pH}$ stability [17]. Our current study aimed to elucidate the lignocellulose-degrading machinery of the fungus in detail and identify novel carbohydrate-active enzymes (CAZymes). We observed some genomic differences between LPH172 and the previously sequenced strain NRRL 8126 . To corroborate genomic CAZyme analysis with transcriptome data, we grew the fungus on four substrates: glucose, Avicel, rice straw, and beechwood xylan. We observed that the fungus relied mainly on LPMOs and canonical cellulases when grown on cellulosic substrates and on hemicellulosic substrates, more conventional hemicellulases were induced together with some LPMOs. Interestingly, we also report co-expression and co-upregulation between LPMOs and other AA enzymes.

\section{Results}

\section{Strain identification}

We previously isolated the T. terrestris strain LPH172 from compost in Northern Vietnam and showed that it could be exploited as an industrially relevant enzyme producer [17]. To confirm the identity of the fungus, first, we used two common fungal housekeeping genes [38] encoding transcription elongation factor $1-\alpha$ and $\beta$-tubulin. The homologous gene sequences used for the identification procedure are listed in Additional file 1. Both housekeeping genes were $100 \%$ identical (e-value 0) to the T. terrestris NRRL 8126 homologues.

Furthermore, we used phylogenetic analysis of closely related species to confirm the strain identity. Three maximum likelihood phylogenetic trees were constructed based on the internal transcribed spacer (ITS-D1D2), RNA polymerase II (RPB2) and $\beta$-tubulin (TUB2) genes. In all three constructed trees, the $T$. terrestris strain LPH172 clustered closely together with four other $T$. terrestris strains (Additional file 1: Figure S1).

Both of these results confirmed the fungus in the current study to be a strain of $T$. terrestris.

\section{Growth on different carbohydrates}

To assess the ability of T. terrestris LPH172 to utilize different carbon sources, the strain was grown on various defined substrates on agar. Growth was measured by the 
diameter and density of mycelia and was compared to a selection of known mesophilic and thermophilic biomass degraders (Fig. 1). T. terrestris LPH172 grew best on starch and xylose, followed by glucose, cellobiose, and beechwood xylan, whereas only modest growth was observed on the cellulosic substrates Avicel and carboxymethyl cellulose (CMC). This finding suggests relatively high activity of amylases, xylanases, and $\beta$-glucosidases. Direct comparison to the previously sequenced $T$. terrestris CBS 117535 (GenBank acc. nr PRJNA249224) showed that LPH172 grew slightly better on most substrates except glucose. Good growth was observed on pectin and inulin (a fructose-based polymer), whereas growth on locust bean gum and guar gum (galactomannans), as well as bark powder was poor (Additional file 2).

\section{Genome characterization \\ General features}

The genome of $T$. terrestris LPH172 was sequenced on a PacBio RS II instrument by GATC Biotech (Constance, Germany); it yielded 5,27,523 reads comprising over 7 billion bases. Table 1 gives an overview of the sequenced T. terrestris LPH172 genome. Its size was determined to be $36.6 \mathrm{Mb}$ and guanine and cytosine (GC) content was $54.80 \%$. Assembly quality, based on basic sequence statistics, was high as revealed by an average contig size (N50) of $3 \mathrm{Mb}$ and N50 read length of 19,832. To assess completeness and integrity of the genome assembly, Benchmarking Universal Single-Copy Orthologs (BUSCO) analysis was performed [39]. Over 98\% of BUSCO genes in the LPH172 genome were complete, indicating excellent assembly integrity. Gene prediction algorithms identified 10,128 protein-coding genes.

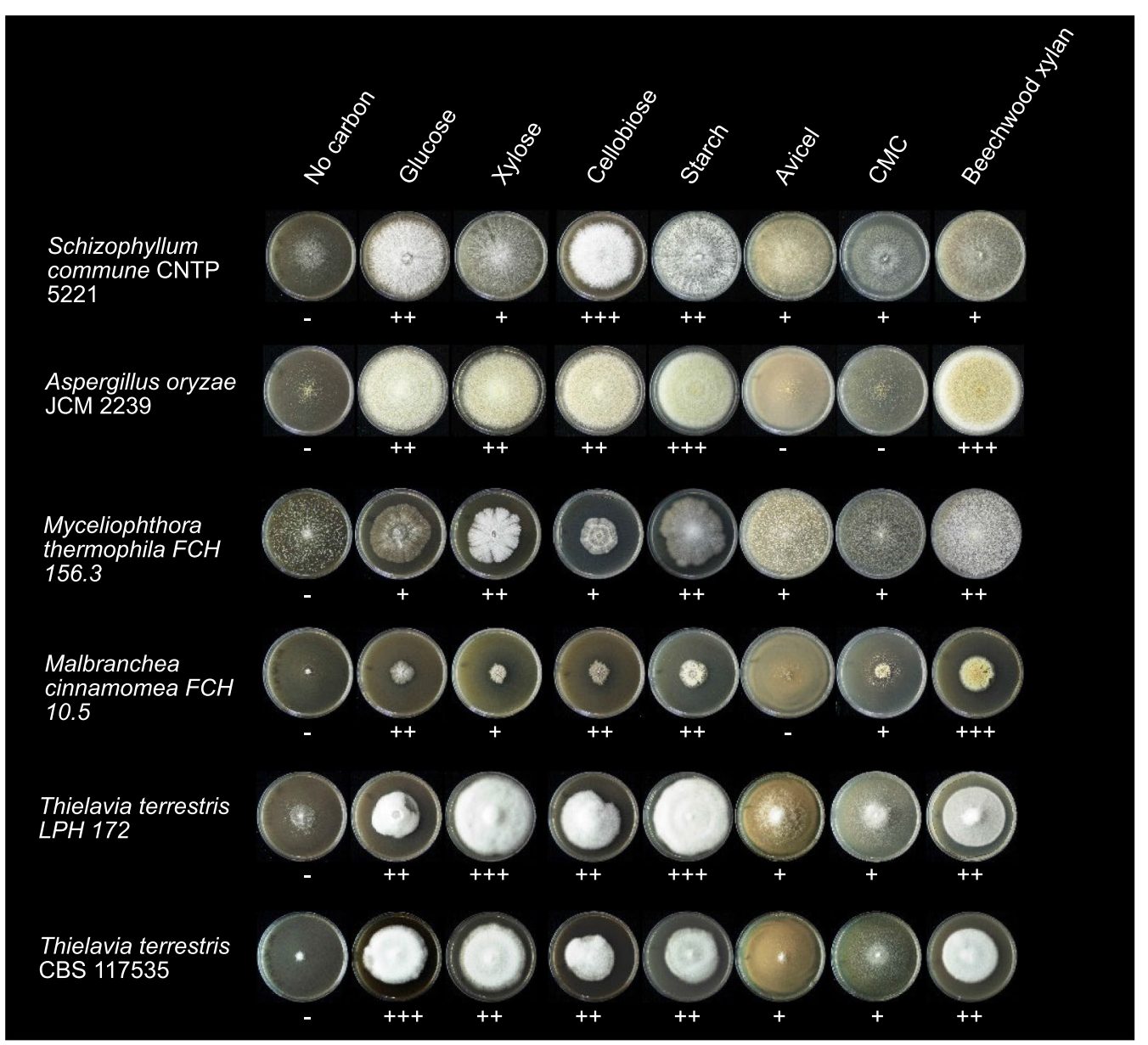

Fig. 1 Growth of T. terrestris LPH172 and other biomass-degrading filamentous fungi on different carbon sources. Seven different carbohydrate substrates at $2 \%(\mathrm{w} / \mathrm{v})$ were used as sole carbon sources for growth on agar plates: monosaccharides (glucose, xylose), disaccharides (cellobiose), and polysaccharides (starch, Avicel, carboxymethyl cellulose-CMC, beechwood xylan). No carbon source was added in the control. The plates were incubated at $30^{\circ} \mathrm{C}$ (S. commune, A. oryzae) or $50^{\circ} \mathrm{C}$ (M. thermophila, M. cinnamomea, T. terrestris) for 2-7 days. Growth was evaluated visually and categorized from - (no growth) to +++ (very good growth), depending on the extent and density of the mycelium 
Table 1 Overview of T. terrestris LPH172 genome as sequenced in this study

\begin{tabular}{ll}
\hline Genome assembly & \\
Number of nucleotides & $36,579,697$ \\
GC content & $54.80 \%$ \\
N50 (bp) & $3,006,457$ \\
Number of protein-coding genes & 10,128 \\
Average gene length (bp) & 1628 \\
Average CDS length (bp) & 1355 \\
Average number of exons per gene & 3 \\
Average exon length (bp) & 460 \\
Longest genes (bp) & 25,297 \\
Longest exons (bp) & 9745 \\
Shortest genes (bp) & 39 \\
Shortest exons (bp) & 3 \\
Fraction of genome covered by & \\
Genes & $45.10 \%$ \\
Exons & $37.80 \%$ \\
Introns & $7.30 \%$ \\
Gene annotation & \\
Genes with functional annotation & 8879 \\
Genes without functional annotation & 1249 \\
Genes annotated (BLASTp, e-value $<1 e^{-6}$ ) & 6114 \\
\hline
\end{tabular}

The size of fungal genomes can vary by orders of magnitude and the average for Ascomycota is $36.91 \mathrm{Mb}[40$, 41]. Table 2 gives a brief overview of the characteristics of the LPH172 genome compared to other industrial and lignocellulose-degrading fungi with varied origin and thermostability. Even though the genome of T. terrestris NRRL 8126 (GenBank assembly nr GCA_000226115), sequenced in 2010, was slightly larger than that of LPH172, our analysis suggested LPH172 contained approximately 200 more genes. This discrepancy, in addition to inherent differences between the two strains, is likely a consequence of ongoing improvements in sequencing and annotation. The genome size of LPH172 was similar to those of other fungi listed in Table 2, as well as to the average Ascomycota genome. The same was true for the average genome GC content. The average gene length in strain LPH172 was $1628 \mathrm{bp}$ and the average coding sequence was $1355 \mathrm{bp}$ (Table 1). On average, three exons per gene were predicted, with exons covering $45.10 \%$ of the genome. $88 \%$ of the genes could be functionally annotated with BLASTp, $69 \%$ of which with high certainty (e-value $<1 \mathrm{e}^{-6}$ ).

\section{Thermostability features}

Although there is no clear consensus on the causes contributing to elevated optimum growth temperatures and thermotolerance in fungi, possible factors include a reduction in genome size [49], higher average GC content in coding regions, and greater $\mathrm{GC}$ content in the third position of codons (GC3 content) [25, 50].

In contrast to the thermophilic Ascomycete Malbranchea cinnamomea [43], the genome of T. terrestris LPH172 was not smaller compared to that of other mesophilic fungi (Table 2). Normalized (gene length) GC content in gene-coding sequences was $63.5 \%$, which was higher than the genome average of $54.80 \%$. When looking only at the subset of genes encoding CAZymes, the average normalized GC content was even higher (64.5\%). Normalized GC3 content in LPH172 was also high, amounting to $80.7 \%$ in coding sequences and $85.7 \%$ in CAZyme-encoding sequences. We also detected gene TT_05393, encoding an unknown protein with 33\% identity (e-value $1.3 \mathrm{e}^{-19}$ ) to the known thermotolerance gene THTA from Aspergillus fumigatus (GenBank: AY560012.1) [51].

Table 2 Genome characteristics in T. terrestris LPH172 and different industrial and lignocellulose-degrading fungi

\begin{tabular}{|c|c|c|c|c|c|c|}
\hline Organism & Phylum & Thermotolerance & Genome (Mb) & Genome GC (\%) & $\begin{array}{l}\text { Number of protein- } \\
\text { coding genes }\end{array}$ & Source \\
\hline A. oryzae & Ascomycota & Mesophilic & 37.9 & 47.2 & 12,030 & [42] \\
\hline M. thermophila & & Thermophilic & 38.7 & 51.4 & 9110 & {$[25]$} \\
\hline M. cinnamomea & & Thermophilic & 25.0 & 49.8 & 9437 & [43] \\
\hline P. anserina & & Mesophilic & 35.0 & 52.0 & 10,588 & [44] \\
\hline T. terrestris $\mathrm{LPH} 172$ & & Thermophilic & 36.6 & 54.8 & 10,128 & This article \\
\hline T. terrestis NRRL 8126 & & Thermophilic & 36.9 & 54.7 & 9813 & {$[25]$} \\
\hline G. trabeum & Basidiomycota & Mesophilic & 37.2 & 52.9 & 11,755 & {$[45]$} \\
\hline S. commune & & Mesophilic & 38.5 & N/A & $13,210^{*}$ & [46] \\
\hline R.pusillus & Zygomycota & Thermophilic & 25.6 & 45.0 & 10,898 & [47] \\
\hline R. oryzae & & Mesophilic & 39.1 & 35.4 & 13,895 & [48] \\
\hline
\end{tabular}

${ }^{*}$ nr of genes 


\section{CAZyme comparison with other fungi}

Plant biomass-degrading and other CAZymes are catalogued into classes, families and subfamilies in the Carbohydrate Active enZymes (CAZy) database (http:// www.cazy.org/) [52].The number of individual CAZyme domains and distribution across different CAZy families in T. terrestris LPH172 was analyzed and compared to other known fungal biomass degraders to assess the propensity for lignocellulose degradation (Table 3). In total, 411 individual CAZy domains were detected in LPH172 using dbCAN2 (HMMER algorithm) (Additional file 3). Most CAZy domains were found to be GHs (201 candidates), with GH18 ( $n=15)$, GH16 $(n=14)$, GH3 $(n=12)$, and GH47 ( $n=10)$ being the most abundant families. There were also 86 glycosyl transferase (GT) domains, 4 polysaccharide lyase (PL) domains, 26 carbohydrate esterase (CE) domains, 83 AA domains, and 11 carbohydrate-binding module (CBM) domains. Compared to strain NRRL 8126, two more GH (one GH16 and one GH47) domains were identified in LPH172, as well as one additional AA12, one GT2, and one CE1 domain (Additional file 3). T. terrestris LPH172 had a relatively low number of PL domains compared to other fungi (Fig. 2), but a larger complement of AA family domains, particularly AA7 $(n=20)$, AA9 $(n=18)$ and AA3 $(n=16)$ (Fig. 3). Five members of AA11 (chitincleaving) LPMO domains were detected in both $T$. terrestris strains, but no AA13 (starch-cleaving LPMOs) or AA14 (xylan-cleaving LPMOs) domains were observed. LPH172 and NRRL 8126 were the only fungi, among the ones selected, presenting an AA16, a recently characterized C1-hydroxylating LPMO [53].

Putative candidates for CAZymes capable of degrading all major lignocellulosic polymers (cellulose, xylan, xyloglucan, (galacto)glucomannan, pectin, and lignin), as well as starch, inulin, and chitin were found. This finding was in line with growth of $T$. terrestris on all these carbon sources (Additional file 2). At least ten putative homologues of known transcription factors directly regulating (hemi)cellulose utilization in ascomycetous fungi [54] were also detected in the genome of T. terrestris LPH172 (Additional file 4).

\section{Transcriptome analysis}

Highly expressed CAZyme-encoding genes during growth on Avicel, rice straw, and beechwood xylan To verify the genome annotation of LPH172 and analyze gene expression, in particular of CAZyme-encoding genes, the transcriptome was analyzed under different growth conditions. The fungus was grown in shake flasks on four substrates-glucose, Avicel, rice straw and beechwood xylan-and total mRNA was extracted and sequenced. Glucose was chosen as reference monosaccharide because its degradation involves a limited number of CAZymes and should, therefore, reflect expression of mostly constitutive CAZyme-encoding genes. Beechwood xylan, comprising a xylan backbone with 4-O-methyl glucuronic acid side groups, was selected to detect CAZymes required for hardwood hemicellulose degradation [43]. Rice straw, which contains approximately $12 \%$ lignin, $38 \%$ cellulose, and $25 \%$ hemicellulose [55] was chosen to represent a complex, heterogeneous substrate requiring a large array of different CAZymes for degradation. Importantly, rice straw has also vast potential as feedstock in biorefinery applications. Finally, Avicel, which is up to $98 \%$ cellulose $[56,57]$, was selected to identify enzymes required to degrade a highly crystalline and recalcitrant cellulosic substrate. Initially, our transcriptome analysis also included corn cob xylan as an arabinoxylan-containing substrate model for cereals. The results were not included

Table 3 Comparison of the number of individual CAZy domains in T. terrestris LPH172 and other filamentous fungi

\begin{tabular}{|c|c|c|c|c|c|c|c|}
\hline & GH & GT & PL & $\mathrm{CE}$ & AA & CBM & Total \\
\hline Aspergillus oryzae & 292 & 92 & 26 & 31 & 96 & 18 & 555 \\
\hline Myceliophthora thermophila & 185 & 75 & 9 & 26 & 66 & 9 & 370 \\
\hline Malbranchea cinnamomea & 118 & 59 & 4 & 14 & 37 & 5 & 237 \\
\hline Thielavia terrestris LPH172 & 201 & 86 & 4 & 26 & 83 & 11 & 411 \\
\hline Thielavia terrestis NRRL 8126 & 199 & 85 & 4 & 25 & 82 & 11 & 406 \\
\hline Gloeophyllum trabeum & 186 & 64 & 9 & 19 & 57 & 6 & 341 \\
\hline Podospora anserina & 215 & 82 & 7 & 45 & 128 & 15 & 492 \\
\hline Schizophyllum commune & 239 & 73 & 17 & 37 & 83 & 16 & 465 \\
\hline Rhizomucor pusillus & 97 & 99 & 2 & 24 & 17 & 2 & 241 \\
\hline Rhizopus oryzae & 90 & 118 & 4 & 31 & 16 & 7 & 266 \\
\hline
\end{tabular}

All CAZy domains were identified using dbCAN2 (HMMER algorithm)

CE10 family domains were excluded

GH glycoside hydrolase, GT glycoside transferase, $A A$ auxiliary activity, $C E$ carbohydrate esterase, $P L$ polysaccharide lyase, $C B M$ carbohydrate-binding module 


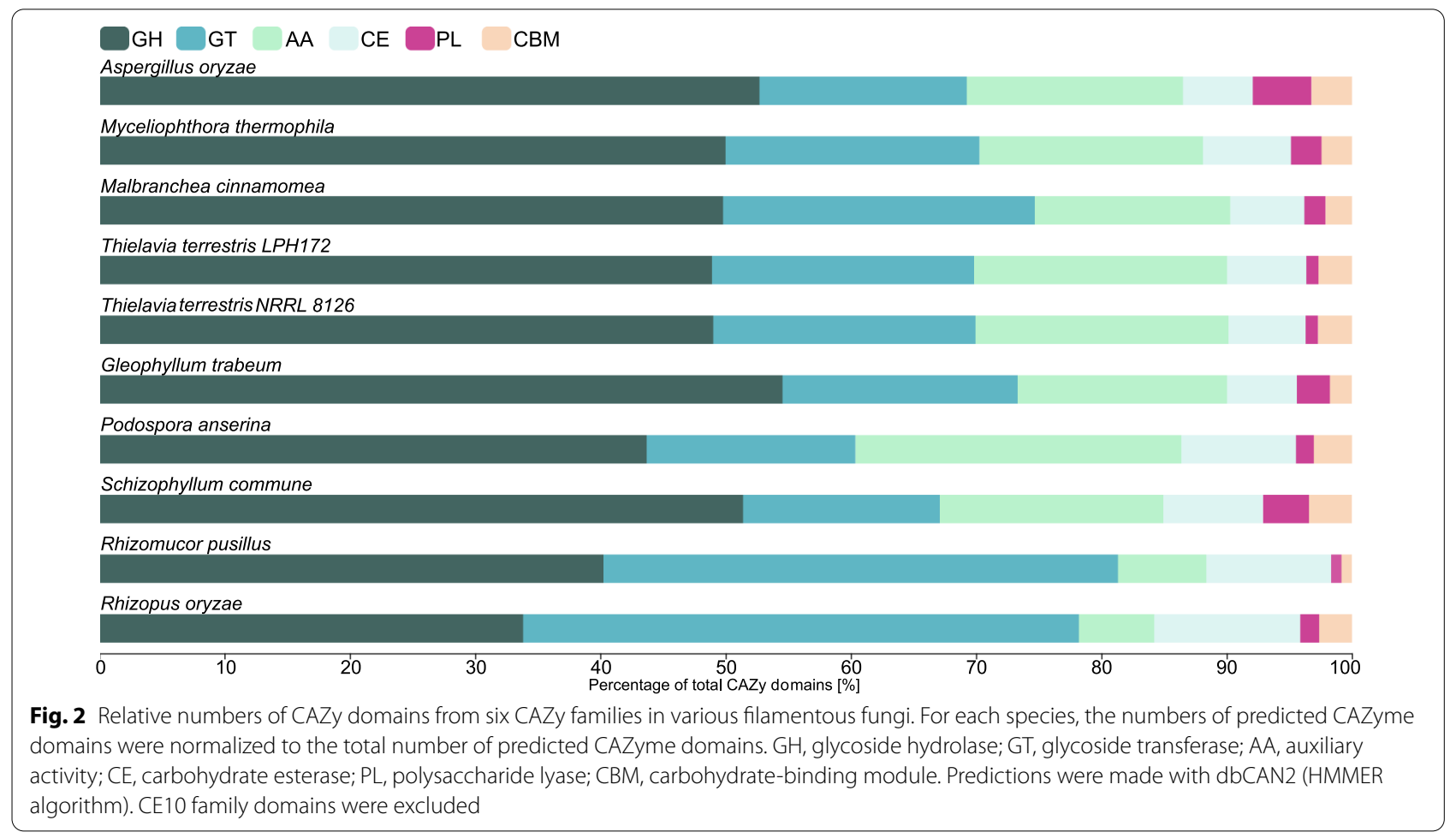

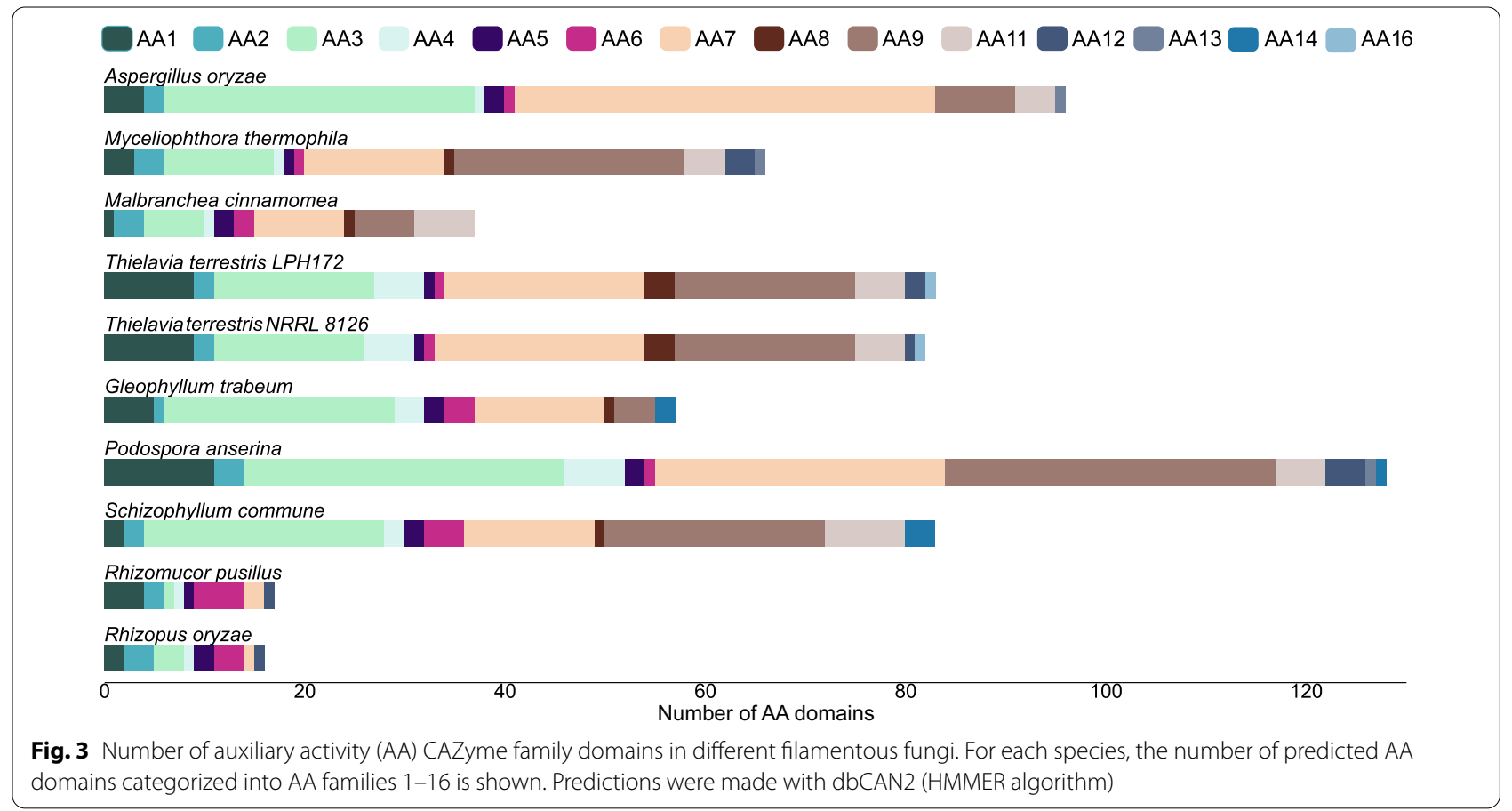

because the purchased corn cob xylan turned out to be composed only of xylo-oligomers [58]. Transcriptome data from RNAseq was used to refine gene annotation through $a b$ initio training with GeneMark v4.3 and an evidence-guided build with MAKER package v3.01.1. Results are summarized in Tables 4-6. 
To identify which CAZyme-encoding genes were the most highly expressed (by transcript abundance) on each chosen substrate, we looked at the top 20 (arbitrary number) candidates under each growth condition, ranked by their average transcripts per million (TPM) value. The complete list of all expressed genes with their TPM values is available in Additional file 5.

Among the top 20 highly expressed CAZyme-encoding genes on Avicel (Table 4) there were in total 11 putative cellulase and LPMO-encoding genes-six cellulases and five LPMOs. These highly expressed cellulase genes included one encoding a putative GH7-CBM1 exoglucanase (TT_05546), a GH6 cellobiohydrolase (TT_06655) and four endoglucanases GH7-CBM1 (TT05797), GH5_5 (TT01019 and TT_00224) and GH45 (TT_09000). All five highly expressed LPMO-encoding genes (TT_07456, TT_08370, TT_04350, TT_06407, TT_01736) belonged to the AA9 family. A gene encoding a CAZyme possibly acting in synergy with LPMOs was also highly expressed-a putative AA3-AA8 cellobiose dehydrogenase encoding gene (TT_04380). Genes encoding five putative xylan-active enzyme genes were also highly abundant on Avicel, encoding a CE5-CBM1 acetylxylan esterase (TT_08166), a feruloyl esterase with

Table 4 Twenty most highly expressed CAZyme-encoding genes during growth of T. terrestris LPH172 on Avicel

\begin{tabular}{lrll}
\hline Transcript ID & TPM & CAZy domain(s) & Putative function \\
\hline TT_05546 & 12001 & GH7-CBM1 & Exoglucanase \\
TT_07456 & 8862 & AA9 & LPMO \\
TT_06655 & 8643 & GH6 & $\begin{array}{l}\text { 1,4- } \beta \text {-D-Glucan cellobiohy- } \\
\text { drolase } \\
\end{array}$ \\
TT_08370 & 8271 & AA9 & LPMO \\
TT_06499 & 6276 & CBM1 & Feruloyl esterase \\
TT_00224 & 4790 & GH5_5 & Endoglucanase \\
TT_05797 & 4654 & GH7-CBM1 & Endoglucanase \\
TT_03075 & 4576 & GH11-CBM1 & Endo-1,4- $\beta$-xylanase \\
TT_04350 & 4537 & AA9-CBM1 & LPMO \\
TT_07210 & 3659 & GH30_7 & Glucosylceramidase \\
TT_08166 & 3512 & CE5-CBM1 & Acetylxylan esterase \\
TT_06407 & 3218 & AA9 & LPMO \\
TT_09000 & 3188 & GH45 & Endoglucanase \\
TT_01736 & 2677 & AA9-CBM1 & LPMO \\
TT_01019 & 2322 & GH5_5 & Endoglucanase \\
TT_04380 & 1599 & AA3_1-AA8 & Cellobiose dehydrogenase \\
TT_01839 & 1429 & GH11 & Endo-1,4- $\beta$-xylanase \\
TT_08161 & 1335 & GH10-CBM1 & Endo-1,4- $\beta$-xylanase \\
TT_03756 & 1104 & GH72 & 1,3- $\beta$-Glucanosyltransferase \\
TT_01913 & 919 & GH132 & Secreted $\beta$-glucosidase \\
\hline TPMvalys & &
\end{tabular}

TPM values indicate average normalized transcript levels from three biological replicates. CAZy domains were predicted by dbCAN2 and functions were annotated by BLASTp search against the UniProt/Swiss-Prot reference dataset a CBM1 domain (TT_06499), two GH11 endo- $\beta$-1,4xylanases (TT_03075 and TT_01839) and a GH10-CBM1 endo- $\beta$-1,4-xylanase (TT_08161). In general, seven of the 20 highly expressed CAZyme genes on Avicel also included a cellulose-binding CBM1 domain.

When the fungus was cultivated on rice straw (Table 5), only one putative cellulase gene (GH131 endoglucanase; TT_01081) was among the top 20 most highly expressed CAZyme-encoding genes. However, five AA9 LPMOs were again highly expressed (TT_07456, TT_08370, TT_04352, TT_06407, TT_03770) - of which TT_07456, TT_08370 and TT_06407 were among the top 20 highly expressed CAZyme genes also on Avicel. None of these putative LPMO-encoding genes expressed during growth on rice straw included a CBM1. For possible xylan backbone degradation, a putative GH11 endo- $\beta-1,4$-xylanase (TT_01839) encoding gene was highly expressed and a putative GH11-CBM1 endo- $\beta$-1,4-xylanase (TT_03075) as well. Both were also highly expressed on Avicel. In addition, a gene encoding a putative CE16 acetyl esterase (TT_06012) possibly cleaving acetyl groups from xylan was among the highly expressed CAZyme genes. Interestingly, on rice straw, genes encoding putative mannosyltransferases belonging to the CAZy families GT15 (TT_07992 and TT_03483), GT32 (TT_08079) and GT2 (TT_05489) were also among the top 20 most highly expressed CAZyme-encoding genes. Moreover, a CE5CBM1 cutinase (TT_08797) was among these top 20 expressed CAZyme genes on rice straw, possibly active on plant cuticle.

When grown on beechwood xylan, curiously, only two putative xylanase encoding genes were among the 20 most highly expressed genes coding for CAZymes (Table 6). These encoded a putative CE5-CBM1 acetylxylan esterase (TT_08166) and a GH11 endo- $\beta$-1,4xylanase (TT_01839), the last one also highly expressed on Avicel and rice straw. No AA9 LPMO-encoding genes were among the 20 most highly expressed CAZyme genes on beechwood xylan, though, a gene encoding a putative AA11 LPMO (TT_09754) was among the top 20 highly expressed genes. Interestingly, several genes encoding putative transferase genes were also highly expressed on beechwood xylan, like on rice straw. These included two genes encoding putative GH72 $\beta$-1,3glucanosyltransferases (TT_03756 and TT_03035) and a gene encoding a putative GT32 mannosyltransferase. Genes coding for putative GH16 glucosidases were also among the top 20 most abundant transcripts on beechwood xylan (TT_08846, TT_09509 and TT_02410). Interestingly, also genes encoding a putative GH37 trehalase (TT_00335) and a putative AA2 catalase-peroxidase (TT_08899) were highly expressed on this hemicellulosic substrate. During growth on both rice straw and 
Table 5 Twenty most highly expressed CAZyme-encoding genes during growth of T. terrestris LPH172 on rice straw

\begin{tabular}{|c|c|c|c|}
\hline Transcript ID & TPM & CAZy domain(s) & Putative function \\
\hline TT_01839 & 8312 & $\mathrm{GH} 11$ & Endo-1,4- $\beta$-xylanase \\
\hline TT_07456 & 963 & AA9 & LPMO \\
\hline TT_08370 & 462 & AA9 & LPMO \\
\hline TT_07992 & 418 & GT15 & Glycolipid 2-a-mannosyltransferase \\
\hline TT_08797 & 400 & CE5 & Cutinase \\
\hline TT_08097 & 276 & GT32 & Initiation-specific a-1,6-mannosyltransferase \\
\hline TT_05010 & 249 & $\mathrm{GH} 25$ & N,O-Diacetylmuramidase \\
\hline TT_01081 & 241 & GH131 & Endoglucanase \\
\hline TT_04352 & 234 & AA9 & LPMO \\
\hline TT_05489 & 178 & GT2 & Dolichol-phosphate mannosyltransferase subunit \\
\hline TT_03075 & 171 & GH11-CBM1 & Endo-1,4- $\beta$-xylanase \\
\hline TT_06407 & 167 & AA9 & LPMO \\
\hline TT_08740 & 166 & CBM52 & Uncharacterized secreted protein \\
\hline TT_09851 & 162 & $\mathrm{GH} 128$ & Alkali-sensitive linkage protein \\
\hline TT_03483 & 158 & GT15 & Mannosyltransferase \\
\hline TT_05929 & 139 & GT31 & $\beta-1,3-N$-Acetylglucosaminyltransferase \\
\hline TT_03756 & 135 & GH72 & $1,3-\beta$-Glucanosyltransferase \\
\hline TT_06012 & 125 & CE16 & Acetylesterase \\
\hline TT_01913 & 119 & GH132 & Secreted $\beta$-glucosidase \\
\hline TT_03770 & 117 & AA9 & LPMO \\
\hline
\end{tabular}

TPM values indicate average normalized transcript levels from three biological replicates. CAZy domains were predicted by dbCAN2 and functions were annotated by BLASTp search against the UniProt/Swiss-Prot reference dataset

Table 6 Twenty most highly expressed CAZyme-encoding genes during growth of T. terrestris LPH172 on beechwood xylan

\begin{tabular}{|c|c|c|c|}
\hline Transcript ID & TPM & CAZy domain(s) & Putative function \\
\hline TT_05010 & 2452 & $\mathrm{GH} 25$ & N,O-Diacetylmuramidase \\
\hline TT_01913 & 1466 & GH132 & Secreted $\beta$-glucosidase \\
\hline TT_03756 & 1366 & $\mathrm{GH} 72$ & 1,3- $\beta$-Glucanosyltransferase \\
\hline TT_09441 & 1359 & CE9 & N-Acetylglucosamine-6-phosphate deacetylase \\
\hline TT_08166 & 1329 & CE5-CBM1 & Acetylxylan esterase \\
\hline TT_08780 & 903 & $\mathrm{GH} 17$ & Glucan endo-1,3- $\beta$-glucosidase \\
\hline TT_03035 & 892 & $\mathrm{GH} 72$ & $1,3-\beta$-Glucanosyltransferase \\
\hline TT_08846 & 743 & GH16-CBM18 & Glycosidase \\
\hline TT_09754 & 736 & AA11 & LPMO \\
\hline TT_01404 & 608 & GH17 & $\beta$-Glucosidase \\
\hline TT_00335 & 516 & GH37 & Trehalase \\
\hline TT_09509 & 487 & GH16 & Glycosidase \\
\hline TT_06210 & 471 & GH30_3 & Endo-1,6- $\beta$-D-glucanase \\
\hline TT_08740 & 469 & CBM52 & Uncharacterized secreted protein \\
\hline TT_00639 & 459 & $\mathrm{GH} 20$ & $\beta$-Hexosaminidase \\
\hline TT_01839 & 419 & GH11 & Endo-1,4- $\beta$-xylanase \\
\hline TT_08097 & 383 & GT32 & Initiation-specific a-1,6-mannosyltransferase \\
\hline TT_08899 & 375 & AA2 & Catalase-peroxidase \\
\hline TT_05769 & 317 & $\mathrm{GH} 128$ & Alkali-sensitive linkage protein \\
\hline TT_02410 & 310 & $\mathrm{GH} 16$ & Extracellular glycosidase \\
\hline
\end{tabular}


beechwood xylan, a gene encoding a putative CAZyme with a CBM52 domain was highly expressed (TT_08740). A putative GH72 $\beta$-1,3-glucanosyltransferase TT_03756 was also among the most highly expresses genes on all three substrates.

Upregulated CAZyme-encoding genes on Avicel, rice straw, and beechwood xylan Absolute gene expression levels do not show the full spectrum of available lignocellulose-degrading enzymes in the organism, because many enzymes are sufficiently active at low concentration and some are constitutively expressed. Therefore, to understand which genes were induced under the tested conditions (Avicel, rice straw or beechwood xylan), we investigated the differential expression of CAZyme-encoding genes with respect to glucose as reference using edgeR [59]. We focused on the transcripts that were significantly highly expressed (i.e., upregulated) compared to glucose in the test conditions $(p \leq 0.05)$ (Additional file 6). A combined list of the top 40 most highly upregulated CAZymeencoding genes on all three substrates is shown in Fig. 4.

On Avicel, the most highly upregulated genes encoded a putative AA9-CBM1 LPMO gene, (TT_01736, $\log _{2}$ fold-change $(\log 2 \mathrm{FC}) 13)$. Other putative AA9 LPMOencoding genes were also highly upregulated: TT08370 and TT07456 had $\log 2 \mathrm{FC} 11$ and TT04350, TT07962 and TT6407 all had $\log 2 \mathrm{FC} 10$. A gene encoding a putative AA3-AA8 cellobiose dehydrogenase was also among the most highly upregulated (TT04380, $\log 2 \mathrm{FC} 9$ ). All AA9 (besides TT07962) and the AA3-AA8 CAZyme-encoding genes presented also very high TPM values, indicating both high upregulation and high expression levels. Other putative CAZyme genes highly upregulated on Avicel encoded mainly GH7 (TT05797 with $\log 2$ FC 8, TT05512 with $\log 2 \mathrm{FC} 12$, TT03032 with $\log 2 \mathrm{FC} 6$, TT05546 with $\log 2 \mathrm{FC} 8$ ), GH5 (TT01019 with $\log 2 \mathrm{FC} 6$, TT03238 with $\log 2 \mathrm{FC} 4$, TT00224 with $\log 2 \mathrm{FC} 9$ ) and GH45 (TT09000 with $\log 2 \mathrm{FC} 5)$ endo- and exo-glucanases. One putative GH6 cellobiohydrolase encoding gene was also highly upregulated (TT06655, $\log 2 \mathrm{FC}$ 8). Interestingly, numerous non-cellulose acting CAZyme-encoding genes were also upregulated on Avicel. These included two feruloyl esterases (TT_06499 with $\log 2 \mathrm{FC} 9$ and TT_09709 with $\log 2 \mathrm{FC}$ 5), five GH10 and GH11 xylanases (TT_06197 with $\log 2 \mathrm{FC} 9$, TT_03205 with $\log 2 \mathrm{FC}$ 5, TT_03075 with $\log 2 \mathrm{FC}$ 7, TT_01839 with $\log 2 \mathrm{FC} 7$, TT_08161 with $\log 2 \mathrm{FC}$ 6), a CE1 acetylxylan esterase (TT_05636 with $\log 2 \mathrm{FC}$ 8), a CE12 rhamnogalacturonan acetylesterase (TT_02368 with $\log 2 \mathrm{FC} 6$ ), a CE16 acetylesterase (TT_06012 with $\log 2 \mathrm{FC}$ 7) and CE5-CBM1 a acetylxylan esterase (TT_08166 with $\log 2 \mathrm{FC}$ 6). Two putative AA7 oxidoreductase encoding genes were highly upregulated on Avicel (TT_03025 with $\log 2 \mathrm{FC} 8$ and TT_06681 with $\log 2 \mathrm{FC}$ 4). Two GH5_7 mannan endo$\beta$-1,4-mannosidase (TT09640 and TT06537) encoding genes were also greatly upregulated on this substrate.

On rice straw, the most highly upregulated genes encoded a putative CE1 acetylxylan esterase (TT_05636 with $\log 2 \mathrm{FC}$ 16), an AA9 LPMO (TT_09068 with $\log 2 \mathrm{FC}$ 13) and a GH11 endo- $\beta$-1,4-xylanase (TT_01839 with $\log 2 \mathrm{FC} 12)$. In general, the set of forty most highly upregulated putative CAZyme-encoding genes on rice straw shared some candidates with Avicel, such as nine AA9 LPMO genes and four hemicellulose-active GH10 and GH11 xylanases, a GH7 endo- $\beta$-1,4-glucanase and CE1, CE5 and CE16 esterases. A putative gene encoding a CBM50 domain (TT_00910) was highly upregulated both on Avicel and rice straw. In addition to the AA9 LPMO genes highly upregulated on Avicel as well, on rice straw two more AA9 genes appeared: TT_03770 and TT_07455 (both with $\log 2 \mathrm{FC}$ 6). Generally, more hemicellulose-active enzyme encoding genes were among the forty highly upregulated genes on rice straw than on Avicel, which is expected with the more complex substrate composition of rice straw. These genes encoded putative GH10 (TT_09033 with $\log 2 \mathrm{FC} 8$ ) and GH11 (TT_02489 with $\log 2 \mathrm{FC} 8$ ) endo- $\beta$-1,4-xylanases, a GH62 $\alpha$-L-arabinofuranosidase (TT_09005 with $\log 2 \mathrm{FC}$ 10), two CE3 esterases (TT_07717 and TT_08970, both with $\log 2 \mathrm{FC}$ 6), a CE1 feruloyl esterase (TT_06092 with

\footnotetext{
(See figure on next page.)

Fig. 4 Combined expression and upregulation values of CAZyme-encoding genes during cultivation on four different substrates. Putative CAZyme-encoding genes involved in biomass degradation were analyzed for their expression levels (TPM, transcripts per million) from three biological replicates, as well as their differential expression (log2FC). The heatmap shows a combination of top forty most highly upregulated CAZyme-encoding genes on three substrates Avicel, rice straw (RS), beechwood xylan (BX) when compared to glucose (Glc). Shading ranges from low expression (light blue) to high expression (magenta). Log2 fold-change (log2FC) shows gene expression during cultivation on Avicel, RS, and BX compared to cultivation on glucose. Shading of upregulated genes (i.e., gene transcripts more abundant on Avicel, RS, and/or BX than on glucose) ranges from light yellow (low upregulation) to dark green (high upregulation). Downregulated genes or genes for which no differential expression was detected or where upregulation was not significant are indicated by blank cells. Only significantly upregulated genes are shown ( $p \leq 0.05)$. All numbers were rounded to the nearest integer. The putative activities of the gene products are based on BLASTp predictions. CAZy domains were analyzed with dbCAN2. The presence of putative signal peptides (SP), predicted by SignalP 4.0, is indicated by a small s. Putative substrates of the CAZymes are: $C$, cellulose; Ch, chitin; GM, glucomannan; L, lignin; $P$, pectin; $S$, starch; $X$, xylan; $X G$, xyloglucan
} 


\begin{tabular}{|c|c|c|c|c|c|c|c|c|c|c|c|}
\hline \multirow{2}{*}{ Transcript ID } & \multirow{2}{*}{ Predicted activity } & \multirow{2}{*}{ SP } & & & & & & & $\log 2 \mathrm{FC}$ & & Putative \\
\hline & & & domain(s) & Avicel & RS & BX & GIc & Avicel & RS & BX & substrate(s) \\
\hline तT 00150 & Laccase & & AA1 & \begin{tabular}{|l|l}
6 \\
\end{tabular} & 22 & 56 & 7 & & 4 & 3 & $\mathrm{~L}$ \\
\hline ПТ 07374 & Multicopper oxidase & & AA1 2 & 9 & 0 & 14 & 3 & 2 & & 2 & $\mathrm{~L}$ \\
\hline Tा 09261 & Laccase & $\mathbf{s}$ & AA1 3 & 3 & 0 & 0 & 0 & 7 & 2 & 3 & L \\
\hline तT 05531 & Catalase peroxidase & $\mathbf{s}$ & AA2 & 10 & 0 & 62 & 15 & & & 2 & \\
\hline Tा 04380 & Cellobiose dehydrogenase & $\mathbf{s}$ & AA3 1-AA8 & 1599 & 4 & 4 & 2 & 9 & 3 & & C \\
\hline तT 08234 & Oxygen-dependent choline dehydrogenase & & AA3 2 & 235 & 2 & 108 & 9 & 5 & & 4 & C \\
\hline Tा 03025 & FAD-linked oxidoreductase & $\mathbf{s}$ & AA7 & 1 & 0 & 0 & 0 & 8 & 5 & & $\mathrm{c}$ \\
\hline Tा 06681 & Oxidoreductase & s & AA7 & 29 & 8 & 2 & 2 & 4 & 4 & & $\mathrm{c}$ \\
\hline Tा 02325 & Cytochrome-domain containing protein & $\mathbf{s}$ & AA8 & 3 & 61 & 0 & 0 & 3 & 10 & & C \\
\hline तT 09190 & Cytochrome-domain containing protein & & AA8 & 7 & 30 & 1 & 7 & & 5 & & C \\
\hline TT 01683 & LPMO & & AA9 & 91 & 6 & 4 & 3 & 5 & 3 & & C \\
\hline Tा 01736 & LPMO & $\mathbf{s}$ & АА9-СBM1 & 2677 & 30 & 20 & 0 & 13 & 9 & 6 & $\mathrm{c}$ \\
\hline Т 03770 & LPMO & & AA9 & 41 & 117 & 4 & 10 & 2 & 6 & & $\mathrm{c}$ \\
\hline तT 04350 & LPMO & $\mathbf{s}$ & AА9-CBM1 & 4537 & 9 & 13 & 6 & 10 & 3 & 1 & C \\
\hline Tा 04352 & LPMO & $\mathbf{s}$ & AA9 & 181 & 234 & 3 & 5 & 5 & 8 & & C \\
\hline ТT 05592 & LPMO & & AA9 & 11 & 0 & 7 & 1 & 4 & 1 & 3 & C \\
\hline ТT 06268 & LPMO & $\mathbf{s}$ & AA9 & 7 & 47 & 4 & 0 & 6 & 11 & 5 & C \\
\hline Tा 06407 & LPMO & & AA9 & 3218 & 167 & 32 & 4 & 10 & 8 & 3 & C \\
\hline Tा 07455 & LPMO & & AA9 & 8 & 7 & 0 & 0 & 4 & 6 & & C \\
\hline ТT 07456 & LPMO & & AA9 & 8862 & 963 & 9 & 4 & 11 & 10 & 1 & C \\
\hline ПT 07962 & LPMO & & AA9 & 60 & 14 & 1 & 0 & 10 & 10 & 3 & C \\
\hline ТT 08370 & LPMO & $\mathbf{s}$ & AA9 & 8271 & 462 & 21 & 3 & 11 & 10 & 3 & C \\
\hline ТT 09068 & LPMO & & AA9 & 0 & 9 & 0 & 0 & 6 & 13 & 3 & C \\
\hline ТT 09080 & LPMO & s & AA9 & 1 & 27 & 1 & 1 & 1 & 8 & & C \\
\hline IT 09453 & PQQ-dependent pyranose dehydrogenase & & AA12 & 31 & 1 & 120 & 4 & 3 & 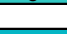 & 5 & \\
\hline$\pi 05636$ & Acetylxylan esterase & $\mathbf{s}$ & CE1 & 2 & 64 & 0 & 0 & 8 & 16 & & $x$ \\
\hline Tा 06092 & Feruloyl esterase & $\mathbf{s}$ & CE1 & 10 & 91 & 6 & 2 & 2 & 8 & 2 & $x$ \\
\hline Tा 02368 & Rhamnogalacturonan acetylesterase & $\mathbf{s}$ & CE12 & 2 & 0 & 1 & 0 & 6 & 6 & 4 & $\mathrm{P}$ \\
\hline Tा 06012 & Acetylesterase & $\mathbf{s}$ & CE16 & 915 & 125 & 8 & 8 & 7 & 6 & & $\mathrm{x}$ \\
\hline П 07717 & Multidomain esterase & $\mathbf{s}$ & CE3 & 5 & 84 & 1 & 6 & & 6 & & $\mathrm{x}$ \\
\hline Tा 08970 & Multidomain esterase & $\mathbf{s}$ & CE3 & 3 & 46 & 1 & 4 & & 6 & & $x$ \\
\hline ПТ 05762 & Acetylxylan esterase & $\mathbf{s}$ & CE5 & 17 & 16 & 44 & 1 & 5 & 7 & 6 & $\mathrm{x}$ \\
\hline ПT 08166 & Acetylxylan esterase & $\mathbf{s}$ & CE5-CBM1 & 3512 & 84 & 1329 & 70 & 6 & 3 & 4 & $\mathrm{x}$ \\
\hline ПТ 08797 & Cutinase & $\mathbf{s}$ & CE5 & 2 & 400 & 6 & 8 & & 8 & & \\
\hline Tा 09441 & $\mathrm{~N}$-acetylglucosamine 6-phosphate deacetylase & & CE9 & 121 & 35 & 1359 & 66 & & 2 & 4 & \\
\hline Tा 06499 & Feruloyl esterase & $\mathbf{s}$ & CBM1 & 6276 & 40 & 10 & 13 & 9 & 4 & & $\mathrm{x}$ \\
\hline तT 04751 & Protein phosphatase 1 regulatory subunit & & CBM21 & 278 & 6 & 218 & 48 & 3 & & 2 & \\
\hline$\pi 00910$ & LysM domain-containing protein & & CBM50 & 71 & 45 & 1 & 0 & 9 & 11 & 2 & $\mathrm{Ch}$ \\
\hline$\pi 06197$ & Endo- $\beta$-1,4-xylanase & $\mathbf{s}$ & GH10 & 245 & 10 & 1 & 0 & 9 & 7 & 2 & $x$ \\
\hline ПТ 08161 & Endo- $\beta$-1,4-xylanase & $\mathbf{s}$ & GH10-CBM1 & 1335 & 14 & 40 & 25 & 6 & 2 & & $\mathrm{x}$ \\
\hline तT 09033 & Endo- $\beta$-1,4-xylanase & $\mathbf{s}$ & GH10 & 2 & 32 & 2 & 1 & 2 & 8 & 1 & $\mathrm{x}$ \\
\hline Tा 01839 & Endo- $\beta$-1,4-xylanase & $\mathbf{s}$ & GH11 & 1429 & 8312 & 419 & 9 & 7 & 12 & 6 & $\mathrm{x}$ \\
\hline Tा 02489 & Endo- $\beta-1,4$-xylanase & s & GH11 & 11 & 30 & 1 & 1 & 4 & 8 & & $x$ \\
\hline Tा 03075 & Endo- $\beta$-1,4-xylanase & $\mathbf{s}$ & GH11-CBM1 & 4576 & 171 & 299 & 33 & 7 & 5 & 3 & $\mathrm{x}$ \\
\hline Tा 03205 & Endo- $\beta$-1,4-xylanase & $\mathbf{s}$ & GH11 & 4 & 40 & 4 & 0 & 5 & 11 & 5 & $\mathrm{x}$ \\
\hline ТT 01081 & Endoglucanase & $\mathbf{s}$ & GH131 & 666 & 241 & 32 & 32 & 4 & 5 & & C \\
\hline ПT 09709 & Feruloyl esterase & s & GH131 & 416 & 5 & 25 & 13 & 5 & 1 & 1 & $\mathrm{x}$ \\
\hline Tा 03813 & Endo-1,3(4)- $\beta$-glucanase & $\mathbf{s}$ & GH16 & 82 & 4 & 104 & 15 & 3 & & 3 & C \\
\hline ПТ 08907 & $\beta$-glucanase & $\mathbf{s}$ & GH16 & 32 & 23 & 139 & 29 & & 2 & 2 & $C, X G$ \\
\hline ПТ 04717 & Chitinase & $\mathbf{s}$ & GH18-CBM18 & 0 & 1 & 13 & 1 & & 2 & 4 & $\mathrm{Ch}$ \\
\hline Tा 05685 & Chitinase & & GH18 & 8 & 17 & 189 & 5 & & 4 & 5 & $\mathrm{Ch}$ \\
\hline Tा 05010 & $\mathrm{~N}, \mathrm{O}$-diacetylmuramidase & $\mathbf{s}$ & GH25 & 356 & 249 & 2452 & 580 & & 1 & 2 & \\
\hline$\pi 06031$ & Rhamnogalacturonase & $\mathbf{s}$ & GH28 & 201 & 7 & 6 & 2 & 7 & 4 & 2 & $\mathrm{P}$ \\
\hline Tा 07104 & Rhamnogalacturonase & $\mathbf{s}$ & GH28 & 0 & 1 & 0 & 0 & & 5 & & $\mathrm{P}$ \\
\hline Tा 07602 & Endopolygalacturonase & s & $\mathrm{GH} 28$ & 2 & 6 & 1 & 0 & 2 & 7 & 2 & $\mathrm{P}$ \\
\hline Tा 07210 & Glycosylceramidase & & GH30 7 & 2650 & 78 & 48 & 7 & 9 & 6 & 3 & \\
\hline Tा 02717 & Trehalase & & GH37 & 119 & 4 & 109 & 25 & 2 & & 2 & \\
\hline Tा 06379 & Endo- $\alpha-1,5$-L-arabinanase & $\mathbf{s}$ & GH43 24 & 120 & 1 & 56 & 10 & 4 & & 3 & $X G, X, P$ \\
\hline ТT 00222 & Arabinan endo- $\alpha-1,5-\mathrm{L}$-arabinosidase & $\mathbf{s}$ & GH43 30 & 4 & 0 & 1 & 0 & 4 & & 2 & $X G, X, P$ \\
\hline Tा 02313 & $\alpha$-L-arabinofuranosidase & & GH43 36 & 53 & 1 & 1 & 2 & 5 & & & $X G, X, P$ \\
\hline तT 09000 & Endo- $\beta$-1,4-glucanase & $\mathbf{s}$ & GH45 & 3188 & 28 & 8 & 96 & 5 & & & C \\
\hline$\pi 00224$ & Endoglucanase & $\mathbf{s}$ & GH5 5 & 4790 & 27 & 7 & 7 & 9 & 4 & & C \\
\hline Tा 01019 & Endo- $\beta-1,4$-glucanase & $\mathbf{s}$ & GH5 5 & 2322 & 26 & 138 & 38 & 6 & 2 & 2 & $\mathrm{c}$ \\
\hline Tा 03238 & Endo- $\beta$-1,4-glucanase & $\mathbf{s}$ & GH5 5 & 347 & 1 & 3 & 9 & 5 & & & C \\
\hline Tा 05879 & Endo- $\beta$-1,4-glucanase & $\mathbf{s}$ & GH5 5 & 31 & 12 & 11 & 1 & 5 & 6 & 3 & $\mathrm{C}$ \\
\hline Tा 06537 & Mannan endo- $\beta-1,4$-mannosidase & & GH5 7 & 311 & 32 & 3 & 2 & 7 & 7 & & GM \\
\hline Tा 09640 & Mannan endo- $\beta-1,4$-mannosidase & $\mathbf{s}$ & GH5 7 & 57 & 6 & 1 & 0 & 9 & 8 & 4 & GM \\
\hline ТТ 01894 & Arabinogalactan endo- $\beta$-1,4-galactanase & $\mathbf{s}$ & GH53 & 173 & 77 & 5 & 6 & 5 & 6 & & $\mathrm{P}$ \\
\hline तT 06655 & 1,4- $\beta$-D-glucan cellobiohydrolase & $\mathbf{s}$ & GH6 & 8643 & 78 & 90 & 48 & 8 & 3 & & $\mathrm{x}$ \\
\hline Tा 09103 & $1,4-\beta$-D-glucan cellobiohydrolase & $\mathbf{s}$ & GH6 & 17 & 3 & 10 & 2 & 3 & 3 & 2 & C \\
\hline Tा 09005 & $a$-L-arabinofuranosidase & s & GH62 & 1 & 13 & 1 & 0 & 4 & 10 & 4 & $X G, X, P$ \\
\hline Tा 03032 & Endoglucanase & $\mathbf{s}$ & $\mathrm{GH} 7$ & 170 & 3 & 7 & 4 & 6 & 2 & & C \\
\hline ТT 05512 & Endo- $\beta-1,4$-glucanase & $\mathbf{s}$ & $\mathrm{GH} 7$ & 164 & 2 & 0 & 0 & 12 & 8 & 3 & C \\
\hline Tा 05546 & Exoglucanase & $\mathbf{s}$ & GH7-CBM1 & 12001 & 38 & 26 & 53 & 8 & 2 & & C \\
\hline ТТ 05797 & Endoglucanase & $\mathbf{s}$ & GH7-CBM1 & 4654 & 20 & 2 & 20 & 8 & 2 & & C \\
\hline Tा 07042 & $1,3-\beta$-glucanosyltransferase & $\mathbf{s}$ & $\mathrm{GH} 72$ & 7 & 33 & 84 & 5 & & 5 & 4 & \\
\hline तT 00146 & Endo-chitosanase & $\mathbf{s}$ & GH75 & 61 & 1 & 19 & 2 & 5 & & 3 & $\mathrm{Ch}$ \\
\hline ТT 03098 & Exo- $\alpha-\mathrm{L}-1,5$-arabinanase & & GH93 & 94 & 23 & 122 & 7 & 4 & 4 & 4 & $P$ \\
\hline
\end{tabular}


$\log 2 \mathrm{FC}$ 8), a CE5 acetylxylan esterase (TT_05762 with $\log 2 \mathrm{FC} 7$ ) and a CE5 cutinase (TT_08797 with $\log 2 \mathrm{FC}$ $8)$. The two putative mannosidase encoding genes highly upregulated on Avicel (TT_09640 with $\log 2 \mathrm{FC} 8$ and TT_06537 with $\log 2 \mathrm{FC} 7$ ) were also highly upregulated on rice straw. Two putative AA7 oxidoreductase encoding genes (TT_06681 with $\log 2 \mathrm{FC} 4$ and TT_03025 with $\log 2 \mathrm{FC} 5$ ), as well as a transcript with an AA8 domain (TT_02325 with $\log 2 \mathrm{FC}$ 10), were also among the top forty highly upregulated genes on rice straw.

On beechwood xylan, upregulation of CAZyme-encoding genes was more subdued with generally lower $\log 2 \mathrm{FC}$ values, and fewer overlaps with the other two substrates were detected. Interestingly, the second most highly upregulated gene encoded a putative AA9-CBM1 LPMO (TT_01736 with $\log$ 2FC 6). Transcripts of six other AA9 LPMOs were also more abundant on beechwood xylan compared to glucose (TT_06268, log2FC 5; TT_07962, $\log 2 \mathrm{FC}$ 3; TT_08370, $\log 2 \mathrm{FC} 3$; TT_06407, $\log 2 \mathrm{FC} 3$; TT_05592, $\log 2 \mathrm{FC} 3$ and TT_09068, $\log 2 \mathrm{FC} 3$ ), although not as strongly upregulated as on other substrates. A gene encoding a putative AA12 PQQ-dependent pyranose dehydrogenase (TT_09453, $\log 2 \mathrm{FC} 5$ ) was also among the top highly upregulated genes on BX. The same applies for a putative AA3_2 dehydrogenase TT_08234 with $\log 2 \mathrm{FC} 4$.

Despite beechwood xylan being a pure xylan substrate, only a fraction of upregulated CAZyme genes were encoding putative xylan-acting enzymes, such as CE5 acetylxylan esterases TT_05762 (log2FC 6), TT_05762 $(\log 2 \mathrm{FC} 6)$ and TT_08166 $(\log 2 \mathrm{FC} 4), \mathrm{GH} 11$ endo-1,4$\beta$-xylanases TT_01839 $(\log 2 \mathrm{FC} 6), \mathrm{TT} \_03205(\log 2 \mathrm{FC} 5)$ and TT_03075 ( $\log 2 \mathrm{FC} 3)$. Interestingly, no GH10 endo1,4- $\beta$-xylanases nor CE1, CE3 or CE16 esterase encoding genes were among the most highly upregulated genes on $\mathrm{BX}$. Two genes encoding enzymes that are active on chitin and possibly involved in fungal cell wall modulation were also highly upregulated on beechwood xylan, such as GH18 chitinases TT_05685 ( $\log 2 \mathrm{FC} 5$ ) and TT_04717 $(\log 2 \mathrm{FC} 4)$. A variety of cellulose-, pectin- and arabinanactive CAZyme-encoding genes were upregulated at low levels $(\log 2 \mathrm{FC} 2-4)$; the same was observed for some genes encoding enzymes typically associated with lignin degradation (Fig. 4, Additional file 6).

\section{Discussion}

The present study sought to explain in detail the enzymatic machinery T. terrestris LPH172 possesses to break down major lignocellulosic polymers based on genome and transcriptome analysis. Specifically, cellulose degradation appeared to rely mostly on LPMOs and some highly expressed canonical cellulases. Compared to other carbon sources, growth on Avicel was poor, yet LPH172 performed better on this substrate than most other fungi (Fig. 1). Poor growth on Avicel could result from lack of cellulase induction or the high crystallinity of Avicel. We noticed similar discrepancy between putative cellulose-degrading genes and poor growth on Avicel in our previous work with M. cinnamomea [43]. Growth discrepancies between the two T. terrestris strains LPH172 and CBS 117535 corroborate previously reported differences in biomass degradation and enzyme production between strains of the same species [17]. It has been reported that two strains of $A$. niger, for example, produced diverse sets of biomass-degrading enzymes, even when grown on the same plant biomass substrates [37, $60]$. This was mainly attributed to the postgenomic and regulatory differences between strains. Differential gene expression analysis helped to suggest the main enzymes involved in degradation of tested substrates (Fig. 4). The range of upregulated CAZyme-encoding genes was perhaps more diverse than expected, with genes encoding mannanases, xylanases, pectinases and lignin-active enzymes being upregulated on all substrates regardless of the presence or absence of the corresponding polymers. Co-regulation of biomass-degrading enzymes or the presence of traces of other polymers could explain induction of these genes. Enzymological studies that compare the activities and activity optima of these enzymes will help determine the function of seemingly redundant enzymes such as the abundantly expressed AA9 LPMO genes.

(Hemi)cellulase encoding genes were highly expressed and upregulated on Avicel, which is a cellulosic substrate and, hence, should not require hemicellulases for degradation. However, this type of unanticipated expression has been shown before in $T$. terrestris, when the cellulosic substrate CMC induced xylanase production [16]. On the other hand, minor amounts of xylan previously reported to be found in Avicel $[56,57]$ could also stimulate xylanase expression. Putative direct (hemi)cellulase regulating transcription factors were analyzed in the genome of LPH172 and the presence of the prominent promiscuous regulator Xyr1 known to affect both cellulases and hemicellulases [61, 62] was detected (Additional file 4). This finding supports the hypothesis of (hemi)cellulase coregulation in this fungus or alternatively, a combination of co-regulation and induction due to the xylan contamination in Avicel.

Since their discovery a decade ago, LPMOs have been studied in several different fungal, bacterial and even insect species, with new families and activities being continuously reported $[53,63,64]$. In T. terrestris LPH172, the transcriptomic data indicate that AA9 LPMOs play a crucial role in cellulose degradation, as six such enzymes were highly upregulated and five were very 
highly expressed during growth on Avicel. Interestingly, on all three polymeric substrates the highest upregulated genes belonged to this family. Several AA9 genes in LPH172 were highly upregulated on rice straw, which contains some cellulose, but also on purified beechwood xylan. We hypothesize that traces of cellulose in the beechwood xylan substrate induce the expression of cellulose-degrading enzymes, or that co-regulation occurs. Alternatively, certain AA9 LPMOs could act on non-cellulosic substrates, including xylan, mannan or xyloglucan, as reported, for instance, for AA9 LPMOs from $M$. cinnamomea [65] and M. thermophila [66] and N. crassa [67]. A clear preference for CBM1-containing genes was shown among the highly expressed and upregulated CAZyme genes on Avicel but also on rice straw, supporting the predicted cellulose-binding character of CBM1 proteins.

Other members of AA CAZy families were also highly expressed and/or significantly upregulated during growth of LPH172 on various substrates. AA3_1-AA8 cellobiose dehydrogenases (CBD) act as reducing agents to fuel LPMO reactions ${ }^{[}\left[23^{\prime} 28^{\prime} 68^{-7} 71^{]]}\right.$. However, not all fungi containing LPMO genes contain supplementary cellobiose dehydrogenase encoding genes [68]. We observed high co-expression and co-upregulation of these enzyme encoding genes on cellulose-containing substrates. The AA3_1-AA8 CBD gene (TT_04380) that was highly coupregulated with several AA9 LPMOs in our study has been shown to act in synergy with a Thermoascus aurantiacus GH61A (AA9) enzyme [28]. Interestingly, on the cellulosic substrates we also noted the upregulation of two AA8 cytochrome domain containing CAZyme genes (TT_02325 and TT_09190) which could also potentially reduce the copper in the active center of LPMOs. However, the electron transfer to these AA8 domains remains unclear. According to Pfam analysis, TT_09190 also contains a putative sugar transporter domain. Moreover, absence of AA8 co-upregulation with AA9 LPMO genes on beechwood xylan might indicate that a different reduction system is utilized on hemicellulosic substrates than on the cellulosic substrates. AA3_2 single-domain flavoenzymes have also been shown to act in synergy as electron donors for LPMOs [72]. Here, we detected coexpression and co-upregulation of an AA3_2 dehydrogenase gene (TT_08234) and AA9 genes on Avicel and beechwood xylan. Other AA CAZymes capable of producing $\mathrm{H}_{2} \mathrm{O}_{2}$, and therefore potentially serving as LPMO co-factors, are AA7 family oxidoreductases. In fact, an AA7 enzyme with a novel oligosaccharide dehydrogenase activity has been recently shown to both transfer electrons to the LPMO active site copper but also produce $\mathrm{H}_{2} \mathrm{O}_{2}$ as a co-substrate of LPMOs [72]. Here, AA7 encoding genes TT_06681 and TT_03025 were upregulated on both Avicel and rice straw; however, their exact roles in biomass degradation have to be elucidated by future studies. Interestingly, out of the 20 AA7 domains in this strain only four were expressed according to our transcriptomic analysis. An AA3 enzyme (TT_07514) that did not fit in the top genes mentioned in our analysis in Fig. 4 but was still significantly upregulated on all substrates, is not yet classified into an AA3 sub-family according to dbCAN. This AA3 encoding gene found to contain two putative GMC-oxidoreductase domains using Pfam analysis, as well as a putative bacterial luciferase-like domain. To our knowledge, such a domain has not been seen before in combination with AA3 domains and may indicate a fifth sub-family of AA3 CAZymes, but further studies are crucial to substantiate this hypothesis.

In general, the elevated number of LPMO-encoding genes in the fungus, together with their high expression and upregulation confirm the importance of (AA9) LPMOs for plant biomass decomposition by T. terrestris and explains why studying the secretomes of this species had such a clear cellulase-boosting effect [19]. The numerous LPMOs in filamentous fungi support the concept of microbial mutualism. According to this concept, some fungi are responsible mainly for LPMO secretion and for attacking crystalline substrate surfaces, thereby making way for others to degrade amorphous polysaccharides and eventually benefitting the whole microbial community $[60,73]$. Such interactions have been documented with regard to the mutually beneficial synthesis of vital growth substances in fungi [74]. Analogously, white rot fungi are known to degrade lignin, whereas brown rotters mainly modify lignin [7], indicating unique specifications for lignocellulose degradation in different filamentous fungi.

Finally, regarding possible genetic factors contributing to fungal thermostability [50], the genome of $T$. terrestris LPH172 revealed high GC content in the coding sequences of all genes and in those encoding CAZymes as well. In addition, the observed high GC3 content could contribute to the thermophilic lifestyle in T. terrestris, as also noted by Berka et al. [25]. Further research is needed to confirm and elucidate the mechanisms of this interesting phenomenon.

\section{Conclusion}

We sequenced and analyzed the genome of a novel T. terrestris strain LPH172. Both genome and transcriptome analyses of the novel thermophilic T. terrestris strain LPH172 revealed in detail the enzymatic machinery used by the fungus to break down lignocellulosic biomass. Using transcriptome data from growth on glucose, Avicel, rice straw, and beechwood xylan we conclude that the fungus relies on an LPMO-centered strategy when grown 
on cellulosic substrates and more on canonical hemicellulases when grown on xylan. The LPMO-focused degradation approach is supported by co-regulation of other AA enzyme encoding genes that likely are expressed as LPMO co-factors. We also detected high GC and GC3 content as possible genomic characteristics contributing to the thermostability of the strain. The present study provides the basis for further biochemical characterization of the lignocellulose-degrading machinery in T. terrestris and filamentous fungi in general. The apparent complementary or redundant nature of certain CAZymes identified in this study needs to be investigated further with enzymological techniques, whereas a more detailed physiological understanding can be achieved with additional transcriptome and proteome studies.

\section{Methods}

\section{Isolation and maintenance of fungi}

Samples containing decaying plant residues (compost, grasses, rice straw, mushroom ground, wood, and soil) were collected from different provinces in Northern Vietnam during 2012-2016. Fungal strains were isolated as described by Thanh et al. [17] by incubation at $50{ }^{\circ} \mathrm{C}$ and under acidic conditions ( $\mathrm{pH} 2.0$ ) on medium containing untreated rice straw as the sole carbon source. After 7-10 days of incubation, fungal colonies were transferred to potato dextrose agar (PDA) plates and purified by hyphal tip culture at $50{ }^{\circ} \mathrm{C}$. The isolates were maintained in PDA slants in a refrigerator at $2-8{ }^{\circ} \mathrm{C}$.

\section{Growth on plates}

Fungal strains were streaked out on solid base medium composed of $4 \mathrm{~g} \mathrm{~L}^{-1} \mathrm{KH}_{2} \mathrm{PO}_{4}, 13.6 \mathrm{~g} \mathrm{~L}^{-1}\left(\mathrm{NH}_{4}\right)_{2} \mathrm{SO}_{4}$, $0.8 \mathrm{~g} \mathrm{~L}^{-1} \mathrm{CaCl}_{2} \cdot \mathrm{H}_{2} \mathrm{O}, 0.6 \mathrm{~g} \mathrm{~L}^{-1} \mathrm{MgSO}_{4} \cdot 7 \mathrm{H}_{2} \mathrm{O}, 0.1 \mathrm{~g} \mathrm{~L}^{-1}$ peptone; $0.1 \mathrm{~g} \mathrm{~L}^{-1}$ yeast extract, $1000 \times$ trace element solution $\left(10 \mathrm{mg} \mathrm{L} \mathrm{FeSO}_{4} \cdot 7 \mathrm{H}_{2} \mathrm{O}, 3.2 \mathrm{mg} \mathrm{L}^{-1}\right.$ $\mathrm{MnSO}_{4} \cdot \mathrm{H}_{2} \mathrm{O}, 2.8 \mathrm{mg} \mathrm{L} \mathrm{ZnSO}_{4} \cdot 7 \mathrm{H}_{2} \mathrm{O}, 4 \mathrm{mg} \mathrm{L}^{-1}$ $\mathrm{CoCl}_{2} \cdot 6 \mathrm{H}_{2} \mathrm{O}, 3.5 \mathrm{mg} \mathrm{L}^{-1} \mathrm{CuSO}_{4} \cdot 5 \mathrm{H}_{2} \mathrm{O}, \mathrm{pH} 5.6$ ), $1 \%$ (w/v) agar, and $2 \%(\mathrm{w} / \mathrm{v})$ of one of the following carbon sources: Avicel, beechwood xylan, starch, guar gum, CMC, citrus pectin, cellobiose, D-glucose, D-xylose, locust bean gum, and inulin from Dahlia tubers or bark powder. Controls contained no carbon source. Plates were incubated at $30{ }^{\circ} \mathrm{C}$ or $50{ }^{\circ} \mathrm{C}$ for $1-7$ days. Cellobiose was supplied by Megazyme. Bark powder was supplied by the Department of Chemistry and Chemical Engineering (Chalmers University of Technology, Gothenburg, Sweden) and contained $10 \%$ dried pine and $90 \%$ dried spruce bark. All other chemicals were supplied by Merck. Fungal strains were received from the collection at the Centre for Industrial Microbiology (Food Industries Research Institute, Hanoi, Vietnam).

\section{DNA and RNA extraction}

To extract genomic DNA, strain LPH172 was grown on a PDA plate for 5 days at $50{ }^{\circ} \mathrm{C}$, the mycelium was divided into six equal parts, and each part was used as inoculum in $100 \mathrm{~mL}$ liquid base medium containing $2 \%$ glucose. Cultures were incubated in 500-mL baffled Erlenmeyer flasks at $50{ }^{\circ} \mathrm{C}$ and $150 \mathrm{rpm}$ for $48 \mathrm{~h}$. The mycelium was harvested by filtering through sterile Miracloth (Merck Millipore) and rinsing extensively with liquid base medium without glucose. After pressing out excessive moisture by hand, the mycelium was snap-frozen in liquid nitrogen and ground to a fine powder in a TissueLyser (Qiagen) at 30-s, 30-Hz intervals with pre-cooled tungsten steel balls. CTAB buffer (2\% CTAB, $100 \mathrm{mM}$ Tris- $\mathrm{HCl}, \mathrm{pH}$ 8.0, $20 \mathrm{mM}$ EDTA, $1.4 \mathrm{M} \mathrm{NaCl}$ ) was immediately added at $10 \mathrm{~mL} / \mathrm{g}_{\text {mycelium }}$, briefly vortexed and the suspension incubated at $57^{\circ} \mathrm{C}$ for $1 \mathrm{~h}$. DNA was purified three times by phenol-chloroform extraction until no interphase was visible, followed by 2-propanol precipitation [75]. The resulting pellet was resuspended in $1 \mathrm{~mL}$ TE buffer (10 mM Tris-HCl, pH 8.0, 1 mM EDTA) and incubated with $200 \mu \mathrm{g} \mathrm{mL}{ }^{-1}$ RNase A (Thermo Fisher Scientific) at $60{ }^{\circ} \mathrm{C}$ for $2 \mathrm{~h}$ to remove residual RNA. After an additional round of phenol-chloroform extraction, the pellet was resuspended in $150 \mu \mathrm{L}$ TE buffer and DNA was further purified with the DNeasy Plant Mini Kit (Qiagen) according to the manufacturer's instructions. Quality of the purified DNA was verified by agarose gel electrophoresis, Nanodrop (Thermo Fisher Scientific), and Qubit Fluorometer (Thermo Fisher Scientific) before genome sequencing.

For RNA extraction, a 100-mL pre-culture on glucose was prepared as described above for DNA extraction. After harvesting and washing the mycelium, this was divided equally between 250-mL baffled Erlenmeyer flasks containing $50 \mathrm{~mL}$ basal liquid medium supplemented with $2 \%$ Avicel, beechwood xylan, rice straw, corn cob xylan or glucose. After 5 days of cultivation at $50{ }^{\circ} \mathrm{C}$ and $150 \mathrm{rpm}$, the mycelium was harvested, frozen, and ground to a powder, as described for DNA extraction. RNA was extracted using TRIzol (Invitrogen) and chloroform, and further purified with the RNAeasy Plant RNA kit (Qiagen) with on-column DNAse digestion. Quality of the purified RNA was checked by agarose gel electrophoresis, Nanodrop (Thermo Fisher Scientific), Qubit Fluorometer (Thermo Fisher Scientific) and Bioanalyzer (Agilent Technologies). High quality RNA for transcriptome sequencing had and OD 260/280 of 1.8-2.0, an OD 260/230 of 2-0-2.2 and an RNA integrity number (RIN) of $\geq 8$. Unless otherwise mentioned, all chemicals were supplied by Merck, except for corn cob xylan (Carbosynth) and rice straw powder (Center for Industrial Microbiology). 


\section{Genome sequencing, assembly, and analysis}

Genome sequencing and assembly was carried out by GATC Biotech (Constance, Germany). According to the company's proprietary protocols, an 8-12-kb library was prepared by DNA fragmentation, size selection, end repair and adapter ligation, primer annealing, and polymerase annealing. Sequencing was performed on a PacBio RS II instrument (raw data output $400 \mathrm{Mb}$ for a genome of $\sim 37 \mathrm{Mb}$ ) with an average read length of $>6000$ bp. De novo assembly of PacBio RS reads was achieved with proprietary GATC Biotech methods optimized for the sequencing technology, read length and type of raw data and included read filtering by length and quality, error correction of long PacBio reads through alignment of short reads ("reads of insert"), assembly of error corrected reads and assembly polishing. Completeness of the genome was assessed with BUSCO (v3.0.2b) against the fungi_odb9 gene dataset (http://buscodev. ezlab.org/datasets/fungiodb9.tar.gz). To analyze GC and GC3 content, seqinr, Biostrings, and sscu R packages were used [76-78].

\section{Transcriptome sequencing, assembly, and analysis}

Transcriptome sequencing was performed by GATC Biotech (Constance, Germany) with the Inview Transcriptome Explore package. Briefly, a randomly primed cDNA library was prepared by purifying poly-A-containing mRNAs, fragmenting, adapter ligation, and PCR amplification. Illumina sequencing with single reads (50 bp) generated 24 million reads per sample that could be mapped to the reference. Quality checks were performed, and all samples achieved a percentage of clean reads $>95 \%$. Assembly and annotation were done by National Bioinformatics Infrastructure Sweden (NBIS). Guided assembly was done with Tophat2 (v2.0.9) and Stringtie (v1.2.2), whereas repeat masking employed the RepeatModeler package (v1.0.8). Ab initio training for annotation was done with GeneMark-ET (v4.3), Augustus, and snap. Gene builds were computed using the MAKER package (v3.01.1), which employed the following software: exonerate (v2.4), Blast+ (v2.2.28), RepeatMasker (v4.0.3), Bioperl (v1.6.922), Augustus (v2.7), tRNAscan (v1.3.1), snap, and GeneMark-ET (v4.3). Functional annotation of genes and transcripts was performed using the translated CDS features of each coding transcript. For each predicted protein sequence, a BLASTp search was performed on the UniProt/Swiss-Prot reference dataset with default parameters (e-value cut-off $=1$, similarity cut-off $=30 \%$ ) to retrieve gene name and protein function. Secreted proteins were predicted using the SignalP 4.0 Server. Genes containing CAZy domains were identified using dbCAN2 (accessed October 2019). Reads were assigned and counted to the genome annotation using featureCounts of the Rsubread package (v2.2.6) in $\mathrm{R}$ and converted to TPMs. Differential gene expression was analyzed using edgeR (v3.30.3) [59] in R with TMMnormalization and removal of reads with less than 1 read per million in all samples.

\section{Supplementary Information}

The online version contains supplementary material available at https://doi. org/10.1186/s13068-021-01975-1.

Additional file 1: Homologous sequences of transcription-elongation factor-alpha and beta-tubulin genes used for identifying the strain as T. terrestris $\mathrm{LPH} 172$ and phylogenetic analysis for the strain identification.

Additional file 2: Growth of T. terrestris LPH172 and other biomassdegrading filamentous fungi on different carbon sources.

Additional file 3: The complete list of putative CAZy domains detected in T. terrestris LPH172 and other filamentous fungi.

Additional file 4: Putative homologues of known transcription factors directly regulating (hemi)cellulose utilization in the genome of T. terrestris LPH172.

Additional file 5: The complete list of all expressed genes on the four tested substrates.

Additional file 6: The complete list of all upregulated putative CAZyme encoding genes on the four tested substrates.

\section{Acknowledgements}

Support from National Bioinformatics Infrastructure Sweden (NBIS) is gratefully acknowledged. We thank Professor Jens Christian Frisvad (Technical University of Denmark) for his help with identifying the strain. We also thank Martin Engqvist and Sinisa Bratulic (Chalmers University of Technology) for their help with the script used for GC(3) content calculations.

\section{Authors' contributions}

SH, LO, VNT conceptualized the study. SH and NTT performed the experiments under supervision of LO, VNT and JL. MT, PR and SH performed the data analyses. MT and SH wrote the manuscript. All authors contributed to results discussions, read and approved the final manuscript. All authors have read and approved the final manuscript.

\section{Funding}

Open access funding provided by Chalmers University of Technology. The collaboration between FIRI and Chalmers was funded by The Swedish Research Council Dnr 348-2014-3523. LO, SH, MT and JL acknowledge the financial support to them from Knut and Alice Wallenberg Foundation and Chalmers Foundation for their activities within Wallenberg Wood Science Center. PR acknowledges the funding from the Novo Nordisk Foundation grant NNF190C0055044.

\section{Availability of data and materials}

All data generated or analyzed during this study are included within the article and as Additional files 1, 2, 3, 4, 5 and 6. The genome assembly has been deposited at DDBJ/EMBL/GenBank under the assembly Accession No. GCA_900343105.1. Transcriptomic data are deposited at the European Nucleotide Archive (ENA) under the Accession No. PRJEB25201 (ERP107096). The strain is deposited in the Food Industries Research Institute (Hanoi, Vietnam) culture collection.

\section{Declarations}

Ethics approval and consent to participate Not applicable. 


\section{Consent for publication \\ Not applicable.}

\section{Competing interests}

The authors declare that they have no competing interests.

\section{Author details}

'Wallenberg Wood Science Centre, Department of Biology and Biological Engineering, Chalmers University of Technology, SE-412 96 Gothenburg, Sweden. ${ }^{2}$ Division of Industrial Biotechnology, Chalmers University of Technology, SE-412 96 Gothenburg, Sweden. ${ }^{3}$ Center for Industrial Microbiology, Food Industries Research Institute, Thanh Xuan, Hanoi, Vietnam.

\section{Received: 14 October 2020 Accepted: 18 May 2021}

Published online: 03 June 2021

\section{References}

1. Hassan SS, Williams GA, Jaiswal AK. Lignocellulosic biorefineries in Europe: Current state and prospects. Trends Biotechnol. 2019;37(3):231-4.

2. Himmel ME, Ding SY, Johnson DK, Adney WS, Nimlos MR, Brady J, et al. Biomass recalcitrance: engineering plants and enzymes for biofuels production. Science. 2007:315(5813):804-7.

3. Van Dyk JS, Pletschke BI. A review of lignocellulose bioconversion using enzymatic hydrolysis and synergistic cooperation between enzymesfactors affecting enzymes, conversion and synergy. Biotechnol Adv. 2012;30(6):1458-80.

4. Klein-Marcuschamer D, Oleskowicz-Popiel P, Simmons BA, Blanch HW. The challenge of enzyme cost in the production of lignocellulosic biofuels. Biotechnol Bioeng. 2012;109(4):1083-7.

5. Adrio JL, Demain AL. Microbial enzymes: tools for biotechnological processes. Biomolecules. 2014;4(1):117-39.

6. Grigoriev IV, Cullen D, Goodwin SB, Hibbett D, Jeffries TW, Kubicek $C P$, et al. Fueling the future with fungal genomics. Mycology. 2011;2(3):192-209.

7. Mäkelä MR, Donofrio N, De Vries RP. Plant biomass degradation by fungi. Fungal Genet Biol. 2014;72:2-9.

8. Patel AK, Singhania RR, Sim SJ, Pandey A. Thermostable cellulases: current status and perspectives. Bioresour Technol. 2019;279:385-92.

9. Atalah J, Cáceres-Moreno P, Espina G, Blamey JM. Thermophiles and the applications of their enzymes as new biocatalysts. Bioresour Technol. 2019;280:478-88.

10. Viikari L, Alapuranen M, Puranen T, Vehmaanperä J, Siika-aho M. Thermostable enzymes in lignocellulose hydrolysis. In: Olsson L, editor. Biofuels. Berlin: Springer; 2007. p. 121-45.

11. Krska D, Larsbrink J. Investigation of a thermostable multi-domain xylanase-glucuronoyl esterase enzyme from Caldicellulosiruptor kristjanssonii incorporating multiple carbohydrate-binding modules. Biotechnol Biofuels. 2020;13(1):1-13.

12. Wang XW, Bai FY, Bensch K, Meijer M, Sun BD, Han YF, et al. Phylogenetic re-evaluation of Thielavia with the introduction of a new family Podosporaceae. Stud Mycol. 2019;93:155-252.

13. Margaritis A, Merchant RFJ. Thermostable cellulases from thermophilic microorganisms. Crit Rev Biotechnol. 1986:4(3):327-67.

14. Durand $\mathrm{H}$. Comparative study of cellulases and hemicellulases from four fungi : mesophiles Trichoderma reesei and Penicillium sp. and thermophiles Thielavia terrestris and Sporotrichum cellophilum. Enzyme Microb Technol. 1984:6:175-80.

15. Yang $S, X u H$, Yan Q, Liu Y, Zhou P, Jiang Z. A low molecular mass cutinase of Thielavia terrestris efficiently hydrolyzes poly(esters). J Ind Microbiol Biotechnol. 2013:40(2):217-26.

16. García-Huante Y, Cayetano-Cruz M, Santiago-Hernández A, Cano-Ramírez C, Marsch-Moreno R, Campos JE, et al. The thermophilic biomass-degrading fungus Thielavia terrestris Co3Bag 1 produces a hyperthermophilic and thermostable $\beta-1,4$-xylanase with exo- and endo-activity. Extremophiles. 2017;21(1):175-86.

17. Thanh VN, Thuy NT, Huong HTT, Hien DD, Hang DTM, Anh DTK, et al. Surveying of acid-tolerant thermophilic lignocellulolytic fungi in Vietnam reveals surprisingly high genetic diversity. Sci Rep. 2019;9(1):1-12.
18. Benoit JB, Yoder JA, Zettler LW, Hobbs HH. Mycoflora of a trogloxenic cave cricket, Hadenoecus cumberlandicus (Orthoptera: Rhaphidophoridae), from two small caves in Northeastern Kentucky. Ann Entomol Soc Am. 2004;97(5):989-93.

19. Merino ST, Cherry J. Progress and challenges in enzyme development for biomass utilization. In: Olsson L, editor. Biofuels. Berlin: Springer; 2007. p. 95-120.

20. Harris PV, Welner D, McFarland KC, Re E, Navarro Poulsen JC, Brown K, et al. Stimulation of lignocellulosic biomass hydrolysis by proteins of glycoside hydrolase family 61: Structure and function of a large, enigmatic family. Biochemistry. 2010;49(15):3305-16.

21. Quinlan RJ, Sweeney MD, Lo Leggio L, Otten H, Poulsen JCN, Johansen $\mathrm{KS}$, et al. Insights into the oxidative degradation of cellulose by a copper metalloenzyme that exploits biomass components. Proc Natl Acad Sci USA. 2011;108(37):15079-84.

22. Westereng B, Ishida T, Vaaje-Kolstad G, Wu M, Eijsink VGH, Igarashi K, et al. The putative endoglucanase PcGH61D from Phanerochaete chrysosporium is a metal-dependent oxidative enzyme that cleaves cellulose. PLOS ONE. 2011. https://doi.org/10.1371/journal.pone.0027807.

23. Phillips CM, Beeson WT, Cate JH, Marletta MA. Cellobiose dehydrogenase and a copper-dependent polysaccharide monooxygenase potentiate cellulose degradation by Neurospora crassa. ACS Chem Biol. 2011;6(12):1399-406.

24. Levasseur A, Drula E, Lombard V, Coutinho PM, Henrissat B. Expansion of the enzymatic repertoire of the CAZy database to integrate auxiliary redox enzymes. Biotechnol Biofuels. 2013;6(1):1-14.

25. Berka RM, Grigoriev IV, Otillar R, Salamov A, Grimwood J, Reid I, et al. Comparative genomic analysis of the thermophilic biomass-degrading fungi Myceliophthora thermophila and Thielavia terrestris. Nat Biotechnol. 2011;29(10):922-7.

26. de Vries RP, Benoit I, Doehlemann G, Kobayashi T, Magnuson JK, Panisko EA, et al. Post-genomic approaches to understanding interactions between fungi and their environment. IMA Fungus. 2011;2(1):81-6.

27. Gilbert M, Yaguchi M, Watson DC, Wong KKY, Breuil C, Saddler JN. A comparison of two xylanases from the thermophilic fungi Thielavia terrestris and Thermoascus crustaceus. Appl Microbiol Biotechnol. 1993;40(4):508-14.

28. Langston JA, Brown K, Xu F, Borch K, Garner A, Sweeney MD. Cloning, expression, and characterization of a cellobiose dehydrogenase from Thielavia terrestris induced under cellulose growth conditions. Biochim Biophys Acta. 2012;1824(6):802-12.

29. Xu H, Yan Q, Duan X, Yang S, Jiang Z. Characterization of an acidic coldadapted cutinase from Thielavia terrestris and its application in flavor ester synthesis. Food Chem. 2015;188:439-45.

30. Woon JS, Mackeen MM, Binsudin AH, Mahadi NM, Illias RM, Murad AM, et al. Production of an oligosaccharide-specific cellobiohydrolase from the thermophilic fungus Thielavia terrestris. Biotechnol Lett. 2016;38(5):825-32.

31. Meng Z, Yang QZ, Wang zhen J, Hou YH. Cloning, characterization, and functional expression of a thermostable type B feruloyl esterase from thermophilic Thielavia terrestris. Appl Biochem Biotechnol. 2019;189(4):1304-17

32. Rodríguez-Mendoza J, Santiago-Hernández A, Alvarez-Zúñiga MT, Gutiérrez-Antón M, Aguilar-Osorio G, Hidalgo-Lara ME. Purification and biochemical characterization of a novel thermophilic exo- $\beta-1,3-$ glucanase from the thermophile biomass-degrading fungus Thielavia terrestris Co3Bag1. Electron J Biotechnol. 2019;41:60-71.

33. Shirke AN, Basore D, Holton S, Su A, Baugh E, Butterfoss GL, et al. Influence of surface charge, binding site residues and glycosylation on Thielavia terrestris cutinase biochemical characteristics. Appl Microbiol Biotechnol. 2016;100(10):4435-46.

34. Banerjee S, Archana A, Satyanarayana T. Xylanolytic activity and xylose utilization by thermophilic molds. Folia Microbiol. 1995;40(3):279-82.

35. Andersen MR, Salazar MP, Schaap PJ, van de Vondervoort PJ, Culley D, Thykaer J. Comparative genomics of citric-acid-producing Aspergillus niger ATCC 1015 versus enzyme-producing CBS 513.88. Genome Res. 2011;21(6):885-97.

36. Xue M, Yang J, Li Z, Hu S, Yao N, Dean RA, et al. Comparative analysis of the genomes of two field isolates of the rice blast fungus Magnaporthe oryzae. PLoS Genet. 2012. https://doi.org/10.1371/journal.pgen.1002869. 
37. de Vries RP, Riley R, Wiebenga A, Aguilar-Osorio G, Amillis S, Uchima CA, et al. Comparative genomics reveals high biological diversity and specific adaptations in the industrially and medically important fungal genus Aspergillus. Genome Biol. 2017;18:1-45.

38. Money NP. Fungal diversity. In: Watkinson SC, Boddy L, Money NP, editors. The Fungi. Cambridge: Academic Press; 2016. p. 1-36.

39. Simão FA, Waterhouse RM, loannidis P, Kriventseva EV, Zdobnov EM. BUSCO: assessing genome assembly and annotation completeness with single-copy orthologs. Bioinformatics. 2015;31(19):3210-2.

40. Mohanta TK, Bae H. The diversity of fungal genome. Biol Proced Online. 2015;17(1):1-9.

41. Stajich JE. Fungal genomes and insights into the evolution of the kingdom. Microbiol Spectr. 2017:5(4):619-33.

42. Kjærbølling I, Vesth T, Frisvad JC, Nybo JL, Theobald S, Kildgaard S, et al. A comparative genomics study of 23 Aspergillus species from section Flavi. Nat Commun. 2020. https://doi.org/10.1038/s41467-019-14051-y.

43. Hüttner S, Nguyen TT, Granchi Z, Chin-A-Woeng T, Ahrén D, Larsbrink J, et al. Combined genome and transcriptome sequencing to investigate the plant cell wall degrading enzyme system in the thermophilic fungus Malbranchea cinnamomea. Biotechnol Biofuels. 2017;10(1):265.

44. Espagne E, Lespinet O, Malagnac F, Da Silva C, Jaillon O, Porcel BM, et al. The genome sequence of the model ascomycete fungus Podospora anserina. Genome Biol. 2008. https://doi.org/10.1186/gb-2008-9-5-r77.

45. Floudas D, Binder M, Riley R, Barry K, Blanchette R, Henrissat B, et al. The Paleozoic origin of enzymatic lignin decomposition reconstructed from 31 fungal genomes. Science. 2012;336:1715-9.

46. Ohm RA, De Jong JF, Lugones LG, Aerts A, Kothe E, Stajich JE, et al. Genome sequence of the model mushroom Schizophyllum commune. Nat Biotechnol. 2010;28(9):957-63.

47. Hüttner S, Granchi Z, Nguyen TT, van Pelt S, Larsbrink J, Thanh VN, et al. Genome sequence of Rhizomucor pusillus FCH 57, a thermophilic zygomycete involved in plant biomass degradation harbouring putative GH9 endoglucanases. Biotechnol Reports. 2018;20:e00279.

48. Ma LJ, Ibrahim AS, Skory C, Grabherr MG, Burger G, Butler M, et al. Genomic analysis of the basal lineage fungus Rhizopus oryzae reveals a whole-genome duplication. PLoS Genet. 2009. https://doi.org/10.1371/ journal.pgen.1000549.

49. Van Noort V, Bradatsch B, Arumugam M, Amlacher S, Bange G, Creevey C, et al. Consistent mutational paths predict eukaryotic thermostability. BMC Evol Biol. 2013. https://doi.org/10.1186/1471-2148-13-7.

50. Salar RK. Origin of thermophily in fungi. In: Salar RK, editor. Thermophilic Fungi. Boca Raton: CRC Press; 2018. p. 29-53.

51. Chang YC, Tsai HF, Karos M, Kwon-Chung KJ. THTA, a thermotolerance gene of Aspergillus fumigatus. Fungal Genet Biol. 2004;41 (9):888-96.

52. Lombard V, Golaconda Ramulu H, Drula E, Coutinho PM, Henrissat B. The carbohydrate-active enzymes database (CAZy) in 2013. Nucleic Acids Res 2014;42(D1):490-5.

53. Filiatrault-Chastel C, Navarro D, Haon M, Grisel S, Herpoël-Gimbert I, Chevret D, et al. AA16, a new lytic polysaccharide monooxygenase family identified in fungal secretomes. Biotechnol Biofuels. 2019;12(1):55.

54. Benocci T, Victoria M, Pontes A, Zhou M, Seiboth B, de Vries RP. Regulators of plant biomass degradation in ascomycetous fungi. Biotechnol Biofuels. 2017:10(152):1-25.

55. Yokoyama S, Matsmura Y. The Asian Biomass Handbook. The Japan Institute of Energy. (2008). p 338

56. van Gool MP. Targeted discovery and functional characterisation of complex-xylan degrading enzymes. [Wageningen]: Wageningen University; 2012

57. Várnai A, Siika-aho M, Viikari L. Restriction of the enzymatic hydrolysis of steam-pretreated spruce by lignin and hemicellulose. Enzyme Microb Technol. 2010;46(3-4):185-93.

58. Mazurkewich S, Poulsen JCN, Lo LL, Larsbrink J. Structural and biochemical studies of the glucuronoyl esterase OtCE15A illuminate its interaction with lignocellulosic components. J Biol Chem. 2019:294(52):19978-87.

59. Robinson MD, McCarthy DJ, Smyth GK. edgeR: A bioconductor package for differential expression analysis of digital gene expression data. Bioinformatics. 2009;26(1):139-40.
60. de Vries RP, Mäkelä MR. Genomic and postgenomic diversity of fungal plant biomass degradation approaches. Trends Microbiol. 2020;28(6):487-99.

61. Klaubauf S, Narang HM, Post H, Zhou M, Brunner K, Mach-Aigner AR, et al. Similar is not the same: differences in the function of the (hemi-) cellulolytic regulator XInR (XIr1/Xyr1) in filamentous fungi. Fungal Genet Biol. 2014;72:73-81.

62. Stricker AR, Grosstessner-Hain K, Würleitner E, Mach RL. Xyr1 (Xylanase Regulator 1) regulates both the hydrolytic enzyme system and D-xylose metabolism in Hypocrea jecorina. Eukaryot Cell. 2006;5(12):2128-37.

63. Sabbadin F, Hemsworth GR, Ciano L, Henrissat B, Dupree P, Tryfona T, et al. An ancient family of lytic polysaccharide monooxygenases with roles in arthropod development and biomass digestion. Nat Commun. 2018. https://doi.org/10.1038/s41467-018-03142-x.

64. Couturier M, Ladevèze S, Sulzenbacher $G$, Ciano L, Fanuel M, Moreau C, et al. Lytic xylan oxidases from wood-decay fungi unlock biomass degradation. Nat Chem Biol. 2018;14(3):306-10.

65. Hüttner S, Várnai A, Petrovic D, Bach CX, Kim Anh DT, Thanh VN, et al. Specific Xylan Activity Revealed for AA9 Lytic Polysaccharide Monooxygenases of the Thermophilic Fungus Malbranchea cinnamomea by Functional Characterization. Appl Environ Microbiol. 2019;85(23):1-13.

66. Frommhagen M, Sforza S, Westphal AH, Visser J, Hinz SWA, Koetsier MJ, et al. Discovery of the combined oxidative cleavage of plant xylan and cellulose by a new fungal polysaccharide monooxygenase. Biotechnol Biofuels. 2015;8(1):4-15.

67. Petrović DM, Várnai A, Dimarogona M, Mathiesen G, Sandgren M, Westereng B, et al. Comparison of three seemingly similar lytic polysaccharide monooxygenases from Neurospora crassa suggests different roles in plant biomass degradation. J Biol Chem. 2019;294(41):15068-81.

68. Kracher D, Scheiblbrandner S, Felice AKG, Breslmayr E, Preims M, Ludwicka K, et al. Extracellular electron transfer systems fuel cellulose oxidative degradation. Science. 2016;352(6289):1098-101.

69. Tan TC, Kracher D, Gandini R, Sygmund C, Kittl R, Haltrich D, et al. Structural basis for cellobiose dehydrogenase action during oxidative cellulose degradation. Nat Commun. 2015;6(1):7542.

70. Courtade G, Wimmer R, Røhr ÅK, Preims M, Felice AKG, Dimarogona M, et al. Interactions of a fungal lytic polysaccharide monooxygenase with $\beta$-glucan substrates and cellobiose dehydrogenase. Proc Natl Acad Sci USA. 2016;113(21):5922-7.

71. Loose JSM, Forsberg Z, Kracher D, Scheiblbrandner S, Ludwig R, Eijsink VGH, et al. Activation of bacterial lytic polysaccharide monooxygenases with cellobiose dehydrogenase. Protein Sci. 2016;25(12):2175-86.

72. Haddad Momeni M, Fredslund F, Bissaro B, Raji O, Vuong TV, Meier S, et al. Discovery of fungal oligosaccharide-oxidising flavo-enzymes with previously unknown substrates, redox-activity profiles and interplay with LPMOs. Nat Commun. 2021;12(1):2132.

73. Boddy L. Interactions between fungi and other microbes. In: Watkinson SC, Boddy L, Money NP, editors. The Fungi. Boston: Academic press; 2016.

74. Kögl F, Fries N. Über den Einfluß von Biotin, Aneurin und Meso-Inosit auf das Wachstum verschiedener Pilzarten. 26. Mitteilung über pflanzliche Wachsturnsstoffe. Hoppe Seylers Z Physiol Chem. 1937;249(2-4):93-110.

75. Sambrook J, Russell DW. Purification of nucleic acids by extraction with phenol:chloroform. Cold Spring Harb Protoc. 2006. https://doi.org/10. 1101/pdb.prot4455.

76. Charif D, Lobry JR. SeginR 1.0-2: A contributed package to the R Project for statistical computing devoted to biological sequences retrieval and analysis. In: Bastolla U, Porto M, Roman HE, Vendruscolo M, editors. Structural Approaches to Sequence Evolution. Berlin: Springer; 2007. p. 207-32.

77. Pagès H, Aboyoun P, Gentleman R, DebRoy S. Biostrings: Efficient manipulation of biological strings. 2020

78. Sun Y. sscu: Strength of Selected Codon Usage. 2020

\section{Publisher's Note}

Springer Nature remains neutral with regard to jurisdictional claims in published maps and institutional affiliations. 